\title{
A checklist of Gasan Mt.: an online platform for virtual specimens
}

\author{
Sungyu YANG ${ }^{\dagger}$, Bo-Mi NAM ${ }^{1 \dagger}$, JuEun JANG ${ }^{2}$, Mi-Jung CHOI ${ }^{2}$, Goya CHOI, \\ Kyong-Sook CHUNG ${ }^{3}$ and Hyeok-Jae $\mathrm{CHOI}^{2 *}$ \\ Herbal Medicine Resources Research Center, Korea Institute of Oriental Medicine, Naju 58245, Korea \\ ${ }^{1}$ International Biological Material Research Center, Korea Research Institute of Bioscience \& Biotechnology, Daejeon 34141, Korea \\ ${ }^{2}$ Department of Biology and Chemistry, Changwon National University, Changwon 51140, Korea \\ ${ }^{3}$ Department of Medicinal Plant Science, Jungwon University, Goesan 28024, Korea \\ (Received 14 August 2020; Revised 16 September 2020; Accepted 10 December 2020)
}

\begin{abstract}
Here, we present an updated checklist based on voucher specimens of vascular plants for Gasan Mt. which is located in Chilgok-gun, Gyeongsangbuk-do, South Korea. The list includes 322 taxa in 212 genera and 81 families of vascular plants, of which 14 are endemic, six are rare plants, 48 are floristic target species, and 14 are considered naturalized in Korea. Based on voucher specimens, Gasan Mt. was the southern limit of Cimicifuga heracleifolia var. bifida Nakai and Iris odaesanensis Y. N. Lee on the Korean peninsula. Moreover, we confirmed a hybrid plant deriving from two Iris parents, I. minutoaurea Makino and I. odaesanensis, in a mountain fortress of Gasan Mt. We also present online databases including 325 voucher specimens deposited at the Korea Institute of Oriental Medicine (KIOM; https://oasis.kiom.re.kr/herblib). This study has great significance as the first floristic study of Korean plants sharing virtual specimens online.
\end{abstract}

Keywords: flora, Gasan Mt., vascular plants, voucher specimens, online platform

생물을 재료로 하는 연구에서 증거표본 확보의 중요성 은 아무리 강조해도 지나침이 없다. 식물의 동정은 연구 자의 주관적 견해 또는 종의 개념에 따라 차이가 있으며 특정 분류군 전공자라도 오동정이 있을 수 있다. 표본은 연구 재료의 불확실성 속에서 동정을 위한 객관적 정보를 제공한다. 또한 환경 변화를 예측할 수 있는 중요한 모니 터링 자료, 식물구계를 재검토할 수 있는 정보와 함께 식 물학을 연구하는 연구자들에게는 분포정보를 제공하고 분류학, 계통학, 형태학, 보존생물학, 생물다양성, 고생물 학 등 다양한 연구 분야에 필수적인 비교 자료가 된다 (Funk, 2003; Suarez and Tsutsui, 2004).

한반도를 대상으로 한 식물상 연구는 한국식물분류학 회지, 한국식물학회지, 자원보존, 한국자원식물학회지 및 기타 대학 학술지 등에서 찾을 수 있다. 한국식물분류학 회지에는 1969년 창간호에 처음으로 Lee (1969)에 의한 식 물상 연구 “대암산의 습원식물”이 출판되었다. 그 후로 약 200편의 식물상 연구가 보고되었으나(Korean Journal of Plant Taxonomy, 2020) 2005년에 와서야 처음으로 증거표
본 목록이 제시되었다. Kim et al. (2005)은 “석회암지대인 덕항산(강원)의 관속식물상과 식물지리" 연구에서 처음 으로 증거표본의 목록을 제시하여 결과의 신뢰성을 높였 으며, 최근 식물분류학회지에 발표된 식물상 연구는 모두 증거표본 목록을 제시하고 있다.

한편 증거표본이 확보된 식물상 연구라 하더라도 오동 정에 의한 부정확한 목록이 제시될 수 있다. Yang et al. (2015)은 울릉도의 관속식물상을 발표하여 울릉도 지역의 식물분포 특성에 대하여 고찰하였다. 이 연구는 증거표본 에 근거한 목록을 제시했지만, 목록에는 부처꽃과 털부처 꽃이 각각 독립된 두 종으로 기록되었고 이는 동일한 종 에 대한 오동정의 결과로 판단된다. 또한 국립수목원에서 는 2003년부터 13년간 한반도 관속식물 분포도 발간사업 을 수행하여 국내 최초로 『한반도 관속식물 종합 분포 도』를 발행하였다(Korea National Arboretum, 2016). 여기 에는 증거표본을 근거로 한 동정 결과를 바탕으로 식물목 록을 제시하였는데, 수염현호색[Corydalis caudata (Lam.) Pers.], 긴산꼬리풀(Veronica longifolia L.), 흑박주가리

\footnotetext{
$\dagger$ These authors contributed equally to this work.

*Author for correspondence: hjchoi1975@changwon.ac.kr
} 
[Cynanchum nipponicum var. glabrum (Nakai) H. Hara] 등은 오동정에 의한 분포로 보인다.

최근 증거표본을 근거로 한 식물상 연구가 활발히 진행 되고 있지만 다른 연구자가 수집한 증거표본을 확인하고 관찰하기에는 많은 제약이 있다. 또한 다수의 식물상 연 구는 주로 대학에서 수행되었으며 이로 인하여 수집된 증 거표본들은 각 학교 내 표본관에 수장되어 있다. 대학의 표본관들은 인력, 수장 공간, 예산 등의 문제로 인하여 표 본 배열 또는 디지털화가 미흡한 상황으로 증거표본 열람 이 어려운 경우가 빈번하다.

우리는 이와 같은 상황을 개선하기 위해 이번 연구에 이 용된 모든 증거표본 디지털 이미지를 온라인으로 공유하 였다. 이 연구에서는 경상북도 칠곡군에 있는 가산의 관 속식물을 조사하고 증거표본을 확보한 후, 증거표본의 정 확한 동정 결과에 의한 식물 목록을 제시함과 동시에 디 지털 이미지와 함께 표본의 상세정보를 웹페이지 링크로 제공하였다.

가산(902 m)은 팔공산 $(1,193 \mathrm{~m})$ 과 함께 낙동정맥의 지맥 인 팔공지맥을 형성하고 위도 $36^{\circ} 02^{\prime} 14.1^{\prime \prime}$, 경도 $128^{\circ} 34^{\prime} 57.6^{\prime \prime}$ 일대에 위치한다. 연중 강수량은 672.2$1,253.0 \mathrm{~mm}$, 연평균 기온 $14^{\circ} \mathrm{C}$, 최저기온 $-10^{\circ} \mathrm{C}$, 최고기온 $38^{\circ} \mathrm{C}$ 정도로 강수량이 적고 한서의 차가 큰 남부내륙형의 기후대에 속한다(Chilgok-gun, 2019). 가산의 식물상은 Kim et al. (1990)에 의하여 105과 350속 616분류군으로 보 고되었으나 식물 목록과 증거표본은 제시하지 않았다. $\mathrm{Kim}$ and Eo (1998)는 전국자연환경조사에서 가산의 관속 식물 279분류군을 보고하였고 Park et al. (2015)은 최근 연 구에서 가산 습지에 분포하는 식물 20 종을 보고하였다. 이처럼 가산을 대상으로 한 종합적이고 객관적인 식물상
연구는 미흡하므로 가산의 정확한 식물 목록을 정리하고 증거표본을 확보하는 작업이 필요한 상황이다.

한편, 식물 목록은 정명과 이명관계에 따라 변동될 수 있으며 오동정을 교정함에 따라 수정될 수 있다. 식물상 연구자들은 동정이 어려운 특정 분류군 전문가들에 의한 재검토 후 정밀한 연구결과를 보고할 수도 있다. 이번 연 구도 오동정과 잘못된 정보를 포함할 수 있다. 하지만 이 번 연구에서 제공하는 증거표본의 상세 정보 페이지에서 해당 분류군의 전문가뿐만 아니라 모든 이용자가 주석 정 보를 추가할 수 있으며 이를 통하여 더욱 정확한 동정 결 과를 제공할 수 있을 것이다.

우리는 이 연구에서 식물상 연구와 증거표본 확보의 중 요성이 다시 한 번 환기되기를 기대한다. 또한 가산의 식 물목록에 대한 증거표본 이미지를 제시하고 이를 통해 교 정 가능한 식물 목록을 제시함으로써 이 지역의 식물분포 학적 특성을 고찰하고 특정 식물의 보전을 위한 정보를 제공하고자 한다.

\section{재료 및 방법}

가산의 관속식물상을 파악하기 위하여 2014년 4월부터 2015년 7월까지 총 10회의 현지 조사에서 증거표본을 수 집하였다(Fig. 1, Table 1). 동정의 정확성을 위하여 꽃, 열 매, 포자 등 생식기관을 포함한 개체만을 수집 대상으로 하였고 수집된 표본은 석엽표본으로 제작하여 한국한의 학연구원 한약표준표본관(KIOM)에 보관하였다.

표본의 동정은 Lee (1980), Lee (1996), Park (2009) 등의 도감을 이용하였고 분류군의 학명은 국가표준식물목록 (Korea National Arboretum, 2017)을 따랐으나 일부 분류군

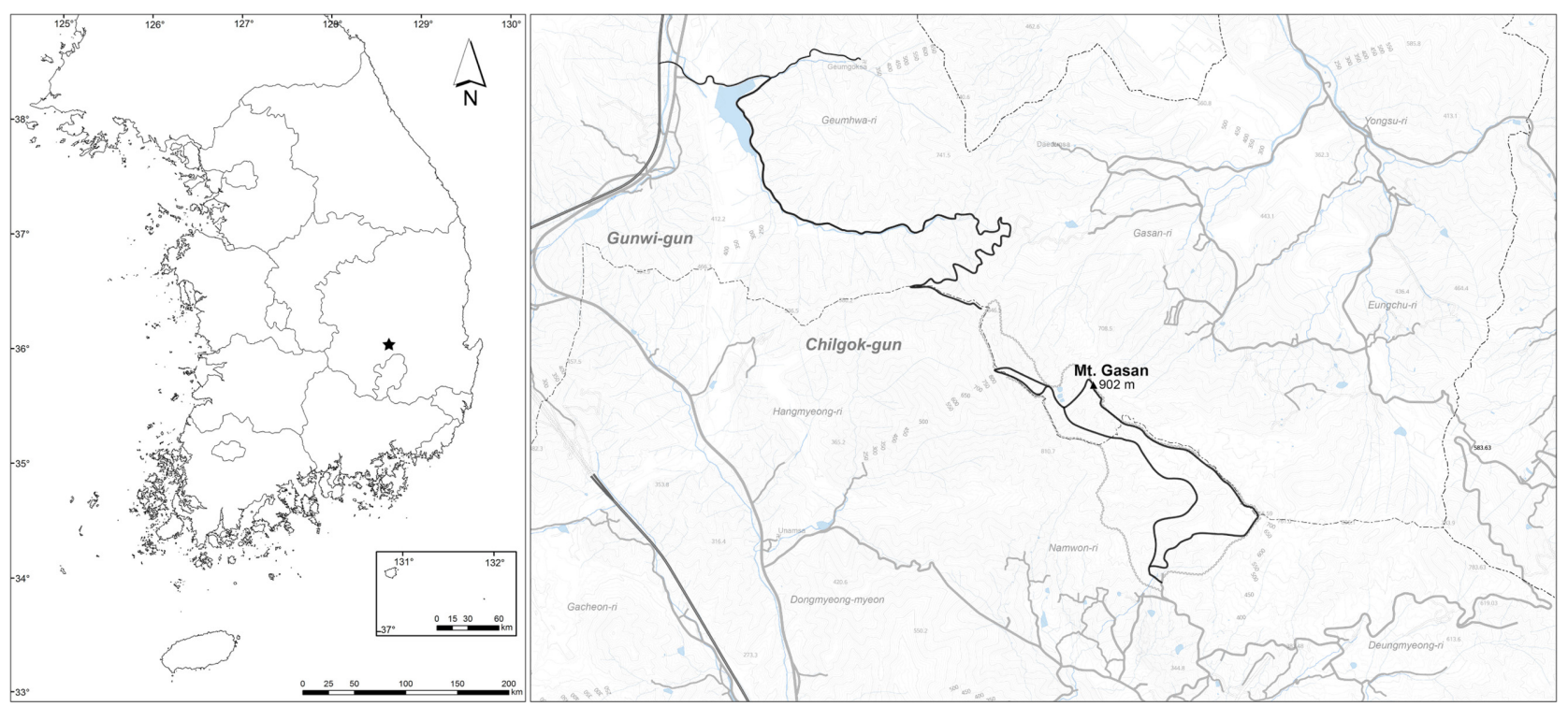

Fig. 1. Floristic routes on Gasan Mt., $\star$ : investigated area (Chilgok-gun, Gyeongsangbuk-do, Korea). 
Table 1. Floristic routes with dates of Gasan Mt.

\begin{tabular}{ccl}
\hline \hline No. & Date & \\
\hline 1 & 23 Apr 2014 & Geumhwaji reservoir $\rightarrow$ Geumgok temple \\
2 & 2 May 2014 & Geumhwaji reservoir $\rightarrow$ Geumgok temple $\rightarrow$ Gyejeong temple \\
3 & 10 May 2014 & $\begin{array}{l}\text { Jinnammun gate } \rightarrow \text { Namporu } \rightarrow \text { Dongmun gate } \rightarrow \text { Jungmun gate } \rightarrow \text { Gasanbawi } \rightarrow \text { Seomun gate } \rightarrow \text { Bukmun } \\
\text { gate } \rightarrow \text { Gasan-bong } \rightarrow \text { Dongmun gate } \rightarrow \text { Bukbawi } \rightarrow \text { Jinnammun gate }\end{array}$ \\
4 & 18 Jun 2014 & Geumhwaji reservoir $\rightarrow$ Moraejae \\
5 & 28 Jul 2014 & Geumhwaji reservoir $\rightarrow$ Moraejae $\rightarrow$ Seomun gate $\rightarrow$ Bukmun gate $\rightarrow$ Gasanbawi $\rightarrow$ Moraejae \\
6 & 30 Sep 2014 & Geumhwaji reservoir $\rightarrow$ Geumgok temple \\
7 & 5 Oct 2014 & Jinnammun gate $\rightarrow$ Namporu $\rightarrow$ Gasanbawi $\rightarrow$ Jungmun gate $\rightarrow$ Namporu $\rightarrow$ Hyeowon temple $\rightarrow$ Jinnammun gate \\
8 & 17 Apr 2015 & Geumhwa camping area $\rightarrow$ Moraejae $\rightarrow$ Seomun gate $\rightarrow$ Bukmon gate \\
9 & 27 Apr 2015 & Geumhwa camping area $\rightarrow$ Moraejae $\rightarrow$ Seomun gate $\rightarrow$ Gasanbawi \\
10 & 28 Jul 2015 & Gasan fortress trekking center $\rightarrow$ Forest trail $\rightarrow$ Bukbawi $\rightarrow$ Dongmun gate \\
\hline
\end{tabular}

은 The Plant List (2013)를 참고하였다. 과명과 분류체계는 양치식물은 PPG I (The Pteridophyte Phylogeny Group, 2016), 피자식물은 APG IV (The Angiosperm Phylogeny Group et al., 2016)에 따라 배열하였고 속과 종은 알파벳순 으로 배열하였다. 주요 식물인 한반도 특산식물(Chung et al., 2017), 희귀식물(Korea National Arboretum, 2008; National Institute of Biological Resources, 2012), 식물구계학 적 특정식물종(National Institute of Ecology, 2018)을 정리하 였으며 귀화식물은 Kang et al. (2020)을 참고하였다. 분포 의 특이성을 보이는 분류군을 파악하기 위해 한반도 관속 식물 분포도(Korea National Arboretum, 2016) 등과 국가생 물종지식정보시스템(http://www.nature.go.kr)의 표본을 확 인하였다. 조사지역의 관속식물 목록은 기존 연구인 $\mathrm{Kim}$ and Eo (1998)의 결과와 이번 연구에서 확인된 결과를 종 합하여 작성하였다(Appendix 1). 특히 이번 연구에서 확인 된 분류군은 결과의 정확성을 높이고 향후 오동정의 교정 을 위하여 증거표본이 확보된 것만을 대상으로 하였으며 식물명과 함께 증거표본번호와 표본의 상세정보를 확인 할 수 있는 웹페이지 링크를 제시하였다.

이번 연구에서 확보된 모든 수집품은 다른 연구자들과의 공유를 위하여 디지털 이미지로 만들어 한국한의학연구원 한약표준표본관 (KIOM) 웹사이트 (http://oasis.kiom.re.kr/ herblib/)에 공유하였다. 디지털 이미지는 제작된 표본을 EPSON 11000XL 평판스캐너(Tokyo, Japan)를 이용하여 $3,443 \times 4,951$ 의 해상도로 스캔하여 만들었다.

\section{결과 및 고찰}

\section{관속식물상}

\section{1. 중거표본에 의한 가산의 관속식물}

이번 연구에서는 가산의 관속식물 81 과 212속 289 종 8 아종 20 변종 5 품종으로 총 322 분류군의 분포를 확인하였 다. 이는 재배식물을 제외한 국내 자생식물 4,171분류군 (Korea National Arboretum, 2017) 중 7.7\%에 해당하며 양치 식물은 7 과 9속 14 분류군, 나자식물 1 과 1 속 1 분류군, 피자 식물 73 과 202속 307 분류군이었다(Table 2). 이 지역의 식 물상에 대한 선행연구는 Kim et al. (1990)와 Kim and Eo (1998)이 있다. Kim et al. (1990)은 가산의 관속식물 616분 류군을 보고하였는데 식물목록을 제시하지 않아서 목록 의 비교 및 분석에 어려움이 있다. 한편 Kim and Eo (1998) 은 증거표본정보와 함께 279분류군의 목록을 보고하였는 데 이 중 이명으로 처리되고 있는 벌엉겅퀴(Circium tanakae Matsum), 취(Aster ageratoides subsp. amplexifolius Kitam.), 큰제비쏙(Artemisia japonica var. manshurica Kom.),

Table 2. Summary data on the flora of Gasan Mt.

\begin{tabular}{lcccccccc}
\hline \hline \multicolumn{1}{c}{ Taxon } & Family & Genus & Species & Subspecies & Variety & Forma & Total & Proportion (\%) \\
\hline Pteridophyta & 7 & 9 & 14 & & & & 14 & 4.3 \\
Gymnospermae & 1 & 1 & 1 & & & & 1 & 0.3 \\
Angiospermae & 73 & 202 & 274 & 8 & 20 & 5 & 307 & 95.4 \\
$\quad$ Dicotyledons & 59 & 163 & 220 & 8 & 18 & 3 & 249 & 77.4 \\
Monocotyledons & 14 & 39 & 54 & & 2 & 2 & 58 & 18.0 \\
\hline \multicolumn{1}{c}{ Total } & 81 & 212 & 289 & 8 & 20 & 5 & 322 & 100 \\
\hline
\end{tabular}


왕잔대(Adenophora tyosenensis Nakai), 민백당나무 (Vibrnum sargentii var. calvescens Rehder), 덤불꼭두서니 (Rubia cordifolia var. sylvatica Maxim.), 개솔나물(Galium verum f. intermedium Nakai), 송금나무(Callicarpa japonica var. taguetii Nakai), 털쥐손이풀(Geranium eriostemon var. typicum Maxim.), 흰싸리(Lespedeza bicolor f. alba Nakai), 왕 팥배나무(Sorbus alnifolia var. macrophylla Nakai), 줄고마리 (Polygonum thynbergii var. stolonifera Nakai), 혹느릅나무 (Ulmus davidiana f. suberosa Nakai)를 제외한 265분류군으 로 정리되었으며 이번 연구에서 확인된 관속식물과 함께 Appendix 1에 목록으로 제시하였다.

가산의 산림식생은 크게 저지대의 일본잎갈나무[Larix kaempferi (Lamb.) Carrière] 조림지와 산림식생의 대부분을 구성하고 있는 산지식생, 정상부의 관목림으로 구분되었 다. 일본잎갈나무 조림지는 주로 금화리와 남원리 일대의 저지대에 넓게 분포하고 있었다. 여기에는 노루발(Pyrola japonica Klenze ex Alef.), 꽃마리[Trigonotis peduncularis (Trevis.) Benth. ex Baker \& S.Moore], 큰개불알풀(Veronica persica Poir.) 등의 초본과 박쥐나무[Alangium platanifolium (Siebold \& Zucc.) Harms], 쥐똥나무(Ligustrum obtusifolium Siebold \& Zucc.)등의 관목이 분포하였다. 산지식생에는 물오리나무[Alnus hirsuta (Spach) Rupr.], 층층나무(Cornus controversa Hemsl.), 곰의말채나무(Cornus macrophylla Wall.), 서어나무[Carpinus laxiflora (Siebold \& Zucc.) Blume] 등의 낙엽활엽수로 대표되었으며 계곡 근처에는 함박꽃나무(Magnolia sieboldii K.Koch)와 쪽동백나무 (Styrax obassis Siebold \& Zucc.)가 주로 관찰되었다. 정상부 의 관목림에는 쇠물푸레나무(Fraxinus sieboldiana Blume), 비목나무(Lindera erythrocarpa Makino), 졸참나무(Quercus serrata Murray) 등의 아교목과 조록싸리(Lespedeza maximowiczii C. K. Schneid.), 국수나무[Stephanandra incisa (Thunb.) Zabel], 조팝나무[Spiraea prunifolia var. simpliciflora (Nakai) Nakai], 병꽃나무[Weigela subsessilis (Nakai) L. H. Bailey] 등의 관목이 우점하는 것을 확인하였다.

\section{2. 특산식물}

가산의 한반도 특산식물은 금오족도리풀[Asarum patens (Yamaki) Yamaki ex Y. N. Lee], 백운산원추리 (Hemerocallis hakuunensis Nakai), 고려엉겅퀴[Cirsium setidens (Dunn) Nakai], 병꽃나무[Weigela subsessilis (Nakai) L. H. Bailey] 등 14 분류군이었으며(Table 3), 한반도 특산식 물 360 분류군의 $3.8 \%$ 에 해당한다(Chung et al., 2017). 특산 식물들은 가산 전 지역에 걸쳐 생육하였으나 몽울토현삼 (Scrophularia cephalantha Nakai)은 북쪽 사면에 드물게 관찰 되었고 흰괭이눈[Chrysosplenium pilosum var. sphaerospermum (A.Terracc.) H. Hara]과 자란초(Ajuga spectabilis Nakai)는 계 곡부에 제한적으로 분포하였다.

\section{3. 희귀식물}

산림청 국립수목원과 환경부 국립생물자원관에서는 희귀식물의 관리를 위하여 세계자연보전연맹의 권고에 따라 목록을 만들어 운영하고 있다. 국립수목원(Korea National Arboretum, 2008)의 기준에 따르면 가산의 식물 중 금 붓꽃(Iris mimutoaurea Makino), 노랑무늬붓꽃(I. odaesanensis Y. N. Lee), 세잎승마[Cimicifuga heracleifolia var. bifida Nakai] 3 분류군이 취약종(vulnerable, $\mathrm{VU}$ )이었고 덩굴꽃마리 [Trigonotis icumae (Maxim.) Makino], 나도개감채[Lloydia triflora (Ledeb.) Baker], 너도바람꽃(Eranthis stellata

Table 3. List of the Korean endemic plants investigated in Gasan Mt.

\begin{tabular}{cll}
\hline \hline No. & \multicolumn{1}{c}{ Family } & \multicolumn{1}{c}{ Taxon } \\
\hline 1 & Aristolochiaceae & Asarum patens (Yamaki) Yamaki ex Y. N. Lee 금오족도리풀 \\
2 & Asphodelaceae & Hemerocallis hakuunensis Nakai 백운산원추리 \\
3 & Asteraceae & Cirsium setidens (Dunn) Nakai 고려엉겅퀴 \\
4 & Caprifoliaceae & Weigela subsessilis (Nakai) L. H. Bailey 병꽃나무 \\
5 & Fabaceae & Vicia chosenensis Ohwi 노랑갈퀴 \\
6 & Iridaceae & Iris odaesanensis Y. N. Lee 노랑무늬붓꽃 \\
7 & Lamiaceae & Ajuga spectabilis Nakai 자란초 \\
8 & Papaveraceae & Corydalis namdoensis B. U. Oh \& J. G. Kim 남도현호색 \\
9 & Paulowniaceae & Paulownia coreana Uyeki 오동나무 \\
10 & Plantaginaceae & Veronica pyrethrina Nakai 큰구와꼬리풀 \\
11 & Ranunculaceae & Cimicifuga heracleifolia var. bifida Nakai 세잎승마 \\
12 & Ranunculaceae & Clematis trichotoma Nakai 할미밀망 \\
13 & Saxifragaceae & Chrysosplenium pilosum var. sphaerospermum (A. Terracc.) H. Hara 흰괭이눈 \\
14 & Scrophulariaceae & Scrophularia cephalantha Nakai 몽울토현삼 \\
\hline
\end{tabular}



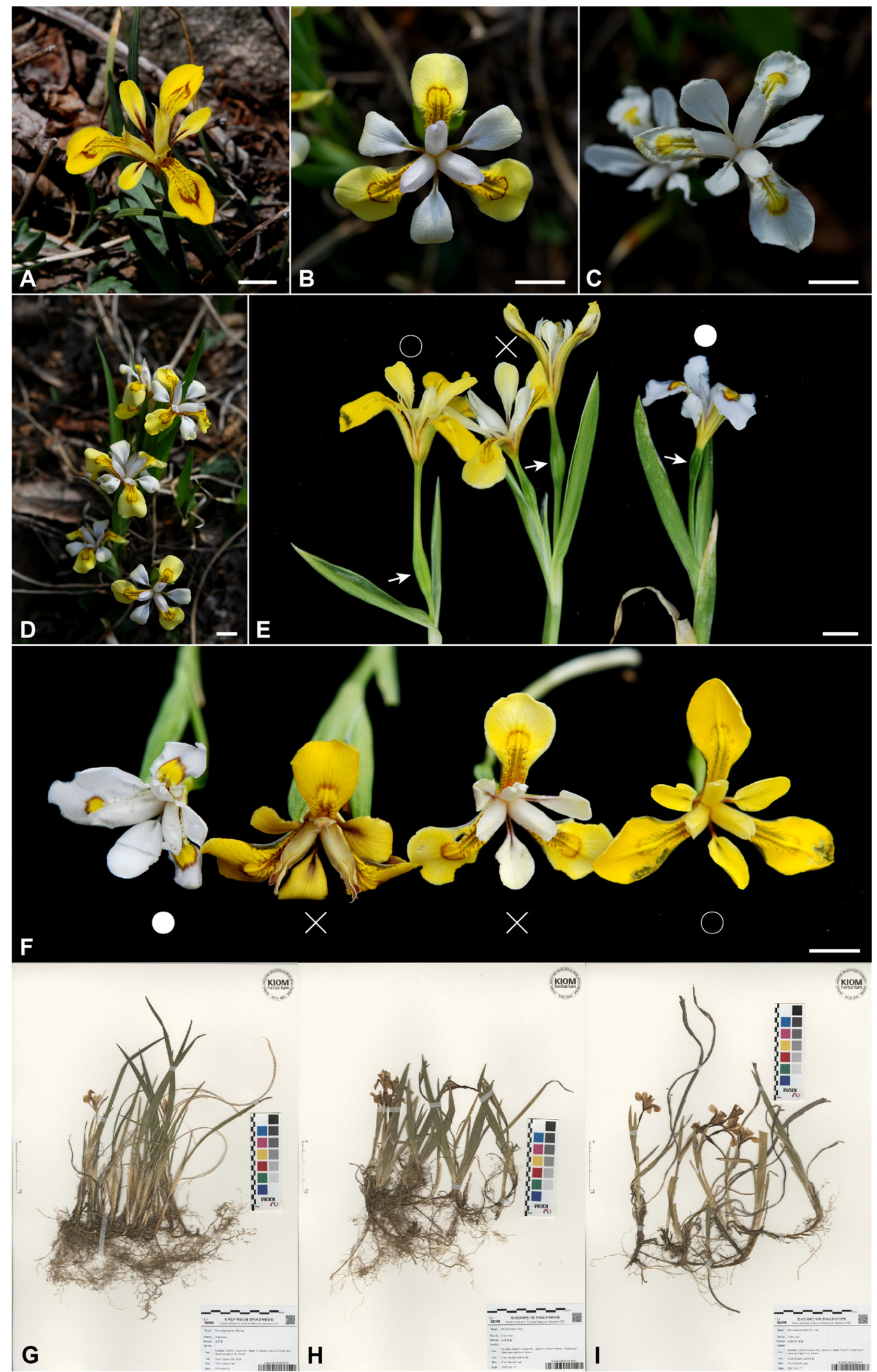

Fig. 2. Photographs of three taxa in the genus Iris and its floral characters. A, G. Iris minutoaurea (०), B, D, H. Iris hybrid plant $(\times)$, C, I. Iris odaesanensis $(\bullet), \mathbf{E}, \mathbf{F}$. flowers of three taxa. Arrows indicates the position of ovary. Scale bars $=1 \mathrm{~cm}$. 
Table 4. List of rare plants investigated in Gasan Mt.

\begin{tabular}{|c|c|c|c|c|}
\hline \multirow{2}{*}{ No. } & \multirow{2}{*}{ Family } & \multirow{2}{*}{ Taxon } & \multicolumn{2}{|c|}{ Criteria } \\
\hline & & & KNA & NIBR \\
\hline 1 & Iridaceae & Iris minutoaurea Makino 금붓꽃 & VU & $\mathrm{LC}$ \\
\hline 2 & Iridaceae & Iris odaesanensis Y. N. Lee 노랑무늬붓꽃 & $\mathrm{VU}$ & $\mathrm{LC}$ \\
\hline 3 & Ranunculaceae & Cimicifuga heracleifolia var. bifida Nakai 세잎승마 & VU & - \\
\hline 4 & Boraginaceae & Trigonotis icumae (Maxim.) Makino 덩굴꽃마리 & LC & $\mathrm{LC}$ \\
\hline 5 & Liliaceae & Lloydia triflora (Ledeb.) Baker 나도개감채 & LC & - \\
\hline 6 & Ranunculaceae & Eranthis stellata Maxim. 너도바람꽃 & LC & - \\
\hline
\end{tabular}

KNA, Korea National Arboretum; NIBR, National Institute of Biological Resources; VU, vulnerable; LC, least concern.

Table 5. List of floristic target species showing category III to V investigated in Gasan Mt.

\begin{tabular}{cllc}
\hline \hline No. & Family & \multicolumn{1}{c}{ Taxon } & Category \\
\hline 1 & Fabaceae & Campylotropis macrocarpa (Bunge) Rehder 꽃싸리 & IV \\
2 & Iridaceae & Iris odaesanensis Y. N. Lee 노랑무늬붓꽃 & Cimicifuga heracleifolia var. bifida Nakai 세잎승마 \\
3 & Ranunculaceae & Sanicula tuberculata Maxim. 애기참반디 & III \\
4 & Apiaceae & Angelica genuflexa Nutt. ex Torr. \& A. Gray 왜천궁 \\
5 & Apiaceae & Brachybotrys paridiformis Maxim. ex Oliv. 당개지치 & Vicia chosenensis Ohwi 노랑갈퀴 \\
6 & Boraginaceae & Veratrum maackii Regel 긴잎여로 \\
7 & Fabaceae & Corydalis namdoensis B. U. Oh \& J. G. Kim 남도현호색 & Veronica daurica Steven 구와꼬리풀 \\
8 & Melanthiaceae & Papaveraceae & Veronica pyrethrina Nakai 큰구와꼬리풀 \\
9 & Plantaginaceae & Lysimachia davurica Ledeb. 좁쌀풀 \\
11 & Plantaginaceae & Eranthis stellata Maxim. 너도바람꽃 \\
12 & Primulaceae & Actaea asiatica H .Hara 노루삼 \\
13 & Ranunculaceae & Aruncus dioicus (Walter) Fernald 눈개승마 \\
14 & Ranunculaceae & Relaginella tamariscina (P.Beauv.) Spring 바위손 \\
15 & Selaginellaceae &
\end{tabular}

Maxim.)의 3분류군이 약관심종(least concerned, LC)으로 확인되었다. 또한 국립생물자원관에서 지정한 적색목록 식물은 약관심종인 금붓꽃, 노랑무늬붓꽃, 덩굴꽃마리 3 분류군이었다(Table 4).

세잎승마는 북서쪽 능선에 드물게 생육하고 있었으며 너도바람꽃, 나도개감채와 덩굴꽃마리는 금곡사 주변 계 곡과 사면부의 자생지를 확인하였다. 금붓꽃과 노랑무늬 붓꽃은 가산산성을 따라 혼생하여 분포하는 것으로 관찰 되었다. Son et al. (2015)은 가산지역의 금붓꽃과 노랑무늬 붓꽃의 자연잡종 현상에 대하여 보고한 바 있다. 가산의 잡종개체군은 기존 관련 문헌에서 노랑붓꽃(Iris koreana Nakai)으로 동정되던 집단으로서 두 모집단인 금붓꽃, 노 랑무늬붓꽃와 혼생하여 분포하였고 화색과 자방의 위치 등의 형질에서 중간형질을 나타냈다(Fig. 2). 한편 잡종개
체군은 금붓꽃, 노랑무늬붓꽃, 노랑붓꽃에 비하여 열매가 결실하지 않는 것으로 관찰되었다.

\section{4. 식물구계학적 특정식물종}

이번 연구에는 가산에 분포하는 특정식물 48 분류군을 확인하였다. IV등급은 꽃싸리[Campylotropis macrocarpa (Bunge) Rehder], 노랑무늬붓꽃, 세잎승마 등 3분류군, III 등급은 애기참반디(Sanicula tuberculata Maxim.), 노랑갈퀴 (Vicia chosenensis Ohwi), 남도현호색(Corydalis namdoensis B. U. Oh \& J. G. Kim), 노루삼(Actaea asiatica H. Hara) 등 13 분류군이 관찰되었다(Table 5). II등급은 개시호 (Bupleurum longiradiatum Turcz.), 큰참나물[Cymopterus melanotilingia (H.Boissieu) C. Y. Yoon], 둥근이질풀 (Geranium koreanum Kom.), 노랑제비꽃[Viola orientalis 
Table 6. List of the naturalized plants investigated in Gasan Mt.

\begin{tabular}{cll}
\hline \hline No. & \multicolumn{1}{c}{ Family } & \multicolumn{1}{c}{ Taxon } \\
\hline 1 & Asteraceae & Ambrosia artemisiifolia L. 돼지풀 \\
2 & Asteraceae & Conyza canadensis (L.) Cronquist 망초 \\
3 & Asteraceae & Symphyotrichum pilosum (Willd.) G. L. Nesom 미국쑥부쟁이 ${ }^{\mathrm{a}}$ \\
4 & Asteraceae & Taraxacum officinale aggr. F. H. Wigg. 서양민들레 \\
5 & Convolvulaceae & Cuscuta pentagona Engelm. 미국실새삼 \\
6 & Fabaceae & Robinia pseudoacacia L. 아까시나무 \\
7 & Onagraceae & Oenothera biennis L. 달맞이꽃 \\
8 & Plantaginaceae & Veronica persica Poir. 큰개불알풀 \\
9 & Poaceae & Dactylis glomerata L. 오리새 \\
10 & Poaceae & Festuca arundinacea Schreb. 큰김의털 \\
11 & Poaceae & Panicum dichotomiflorum Michx. 미국개기장 \\
12 & Polygonaceae & Rumex acetosella L. 애기수영a \\
13 & Polygonaceae & Rumex obtusifolius L. 돌소리쟁이 \\
14 & Polygonaceae & Persicaria orientalis (L.) Spach 털여뀌 \\
\hline
\end{tabular}

${ }^{\mathrm{a}}$ Ecosystem Disturbance Wildplants.

(Maxim.) W. Becker], 돌양지꽃[Potentilla ancistrifolia var. dickinsii (Franch. \& Sav.) Koidz.] 등 17분류군, I등급은 고려 엉겅퀴[Cirsium setidens (Dunn) Nakai], 투구꽃(Aconitum jaluense Kom.), 대극(Euphorbia pekinensis Rupr.) 등 15분류 군이 확인되었다(Appendix 1). 한편 $\mathrm{IV}$ 등급종인 등 [Wisteria floribunda (Willd.) DC.]은 식재분포하고 있었으 므로 특정식물종 목록에서 제외하였다.

\section{5. 귀화식물}

조사지역에 유입된 귀화식물은 돼지풀(Ambrosia artemisiifolia L.), 미국실새삼(Cuscuta pentagona Engelm.), 달맞이꽃(Oenothera biennis L.), 큰개불알풀(Veronica persica Poir.), 오리새(Dactylis glomerata L.) 등 14분류군으 로 확인되었다(Table 6). 조사지역의 도시화지수 (Urbanization Index: 귀화식물의 종수/남한 귀화식물 총 종 수 $\times 100=14 / 427 \times 100)$ 은 $3.2 \%$, 귀화율(Naturalized Index: 귀 화식물의 종수/출현식물의 총 종수 $\times 100=14 / 322 \times 100)$ 은 $4.3 \%$ 이었다. 귀화식물의 비율은 비교적 낮은 수준이었지 만 야영장과 주차장 주변을 중심으로 한 귀화식물의 확산 이 가속화되고 있어 주의 깊은 관찰 및 관리가 필요할 것 으로 판단된다. 특히 생태계교란 야생식물인 돼지풀과 미 국쑥부쟁이[Symphyotrichum pilosum (Willd.) G. L. Nesom], 애기수영(Rumex acetosella L.)이 진남문 주차장 일대와 금 화지 주변에 분포하는 것으로 확인되었으며 이들에 대한 정확한 분포 현황과 확산 방지를 대책이 필요하다. 특히 미국쑥부쟁이와 애기수영은 가산산성 동문 근처에도 관 찰되었으며 산림 내부로의 침투가 우려되므로 적극적인
방제 작업 등이 요구된다.

\section{6. 분포특이종}

가산 또는 이 일대 식물의 분포 특성을 파악하기 위하여 분포 특이성을 나타내는 분류군들을 조사하였다. 세잎승 마는 Nakai (1909)에 의해 변종으로 발표되었으며 Actaea 속에 포함하여 종 수준에서 다루기도 한다(Compton et al., 1998). 주로 강원도와 경북의 태백산맥을 따라 분포하며 통영에도 분포한다(Lee et al., 2000) (Fig. 3A). 노랑무늬붓 꽃은 Lee (1974)에 의해 오대산에서 처음 발견되었으며 토 함산, 보현산, 주왕산, 치악산, 소백산, 태백산 등 강원도와 경상북도의 산지에 자라는 특산식물로 알려져 있다. Flora of China (Zhao et al., 2000)에서는 길림성의 분포를 기록하 고 있으며 특산식물로서의 지위 확인을 위한 추가 연구가 필요하다. 가산.토함산 일대가 이 종 분포의 남방한계인 것 으로 판단된다(Fig. $3 \mathrm{~B})$. 몽울토현삼은 경남 통영 미륵산에 서 처음으로 채집되어 신종으로 발표되었다(Nakai, 1938). 이후 여러 연구자에 의하여 큰개현삼(Scrophularia kakudensis Franch.)의 이명으로 다루었으나(Han et al., 2009; Jang, 2010), Jang et al. (2011)은 개화기 및 형태형질의 차이 를 인식하여 큰개현삼과 독립된 종으로 처리하였다. 표본 검토 결과 본 종은 일월산, 주왕산 등 태백산맥을 따라 통 영 미륵산까지 분포하는 것으로 확인되었다(Fig. $3 \mathrm{C}$ ).

이 분류군들의 특징은 주로 태백산맥을 따라 분포하는 특성을 보이며 가산과 팔공산 일대가 경상도 내륙지역의 남북을 잇는 중요한 지역임을 알 수 있다. 이들 분류군은 조사지역 일대의 분포특성뿐만 아니라 한반도 전체의 분 


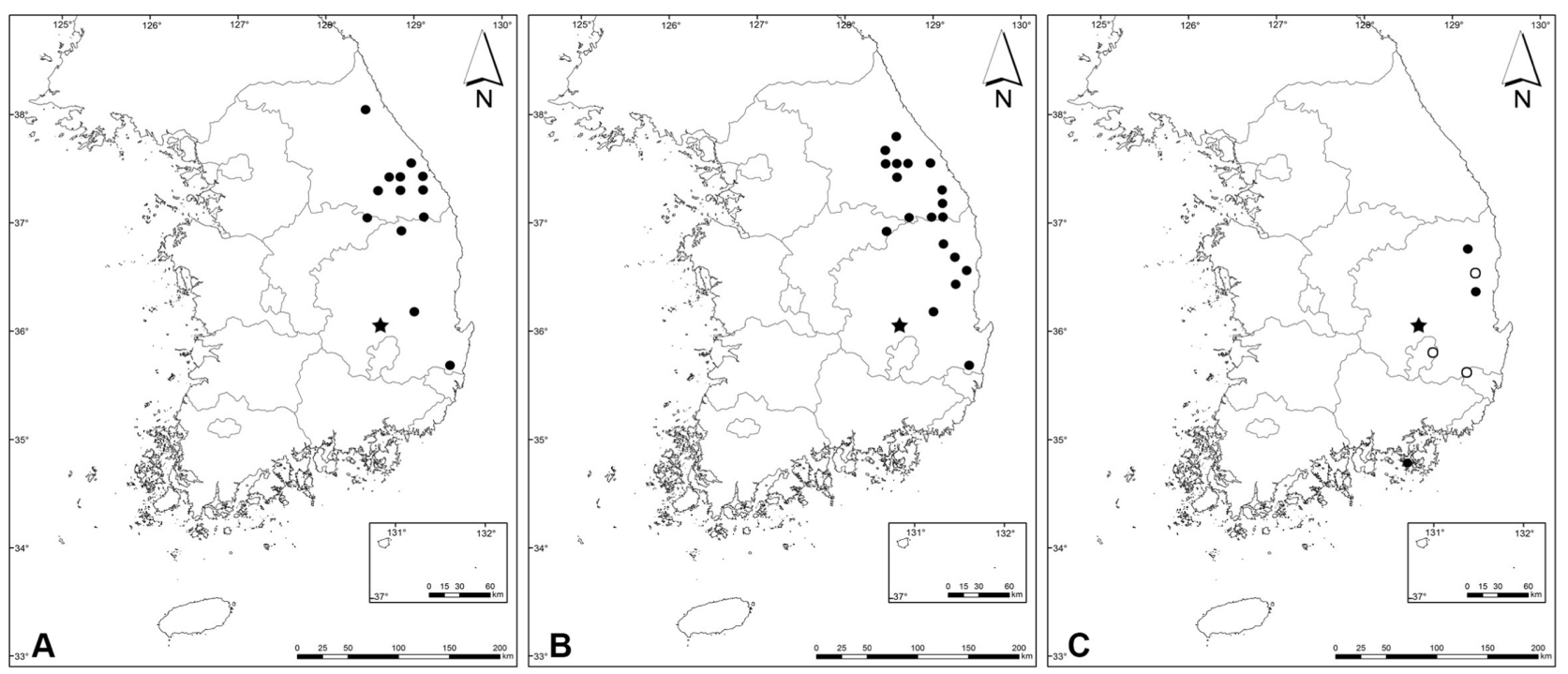

Fig. 3. Maps of three taxa which have unique distribution in Korea. A. Cimicifuga heracleifolia var. bifida Nakai, B. Iris odaesanensis Y. N. Lee, C. Scrophularia cephalantha Nakai. Maps were modified from Korea National Arboretum (2016). ^, confirmed in this study; •, previously recorded distribution; $\circ$, additionally indentified specimens from Korea Biodiversity Information System (http:// www.nature.go.kr) (S. cephalantha: KBNA200102121066 [Samui-ri, Yeongyang-gun, 4 Jun 1972, S. Y. Oh 3909, KNU], KBNA200408262137 [Unmun-ryeong, Cheongdo-gun, 9 Jun 2001, Yang et al., s.n. KNU], KUSA200408182038 [Palgong-san, Daegu-si, 24 May 1998, Kim et al. 24316, YNUH]).
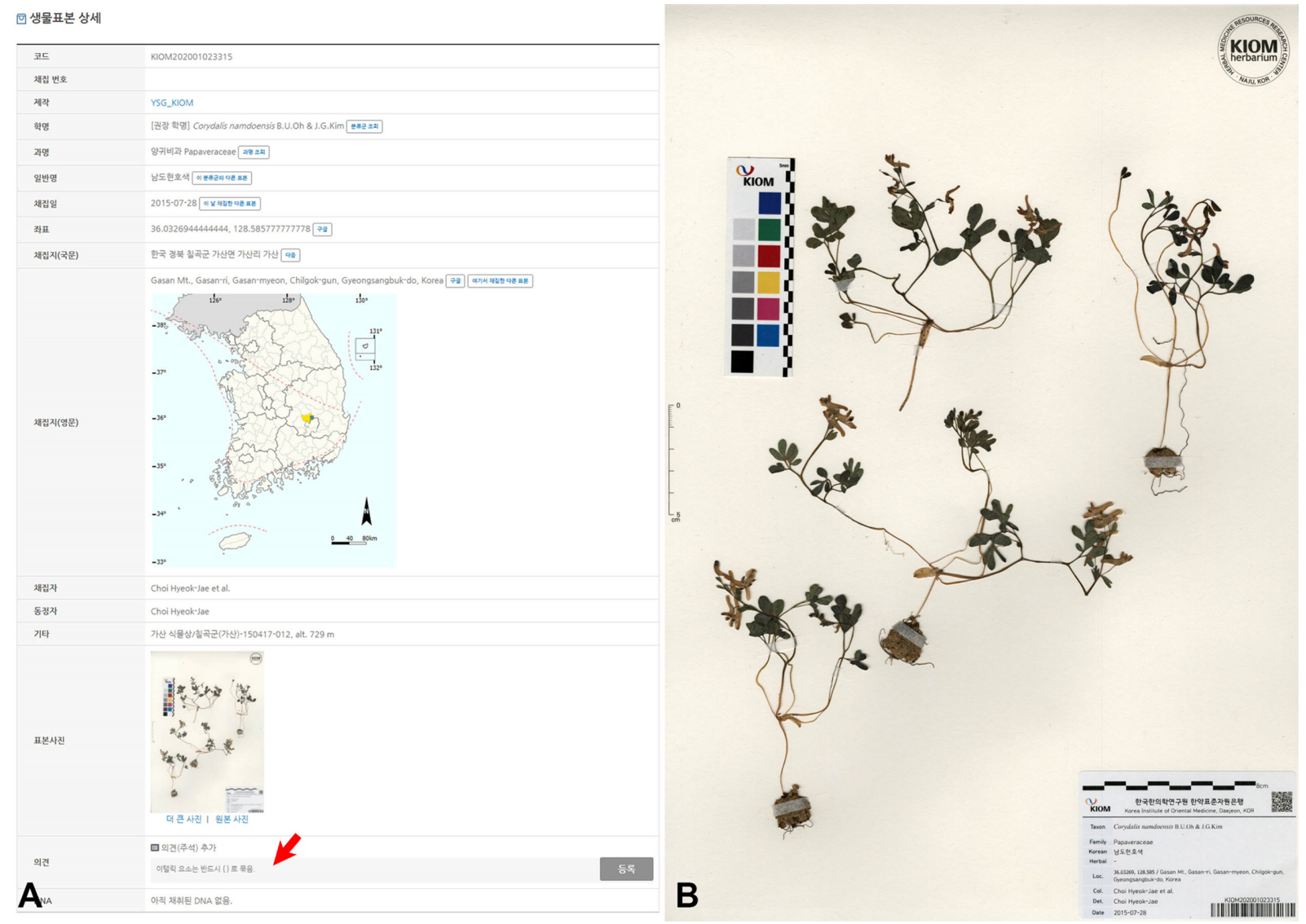

Fig. 4. KIOM databases with metadata, map, and herbarium specimen image (A), and a scanned specimen image of Corydalis namdoensis (B). A arrow displays the annotation function. 
포학적 논의를 위한 높은 학술적 가치를 갖는 분류군들이 므로 주변 지역의 정밀한 자생지 조사가 필요하다.

\section{식물상 연구와 증거표본의 중요성 및 온라인 플랫폼 을 이용한 표본 정보 공유}

증거표본 확보의 중요성은 여러 학자들에 의하여 강조 되어 왔다(Funk, 2003; Suarez and Tsutsui, 2004). 국내에서 는 $\operatorname{Im}$ (1999)이 식물상 연구와 증거표본 확보의 중요성에 대하여 강조하였고 한국식물분류학회는 식물상 연구의 투고원칙에서 “증거표본 정보(collection number 및 표본 소장처)"를 반드시 명시하여야 한다고 규정하고 있다 (Korean Journal of Plant Taxonomy, 2020).

2020년 한국식물분류학회지에는 Sun et al. (2020), Hwang et al. (2020), Park et al. (2020), Kim et al. (2020) 등 에 의한 식물상 연구가 출판되었다. 이 연구들 모두 의미 있는 분석 결과와 함께 증거표본 정보를 제시하고 있지만 다른 연구자들이 증거표본을 직접 확인하기에는 여전히 많은 시간적, 공간적 제약에 놓이게 된다.

따라서 본 연구에서는 기존 식물상 연구들의 한계를 개선하고자 수집된 모든 증거표본을 디지털 이미지로 만들어 수집 정보와 함께 웹페이지 링크로 제시하였다. 가산에 자생하는 관속식물 322 분류군 325 점을 확보하 였고 한국한의학연구원 한약표준표본관 웹사이트 https://oasis.kiom.re.kr/herblib/ [id: flora, password: flora123]에 공유하였다. Appendix 1의 표본번호를 클릭하 여 해당 표본을 직접 확인할 수 있으며 표본에 대한 의견 을 추가하여 오동정을 교정할 수 있다(Fig. 4). 이 연구는 국내 식물상 연구 분야에서 최초로 증거표본에 대한 디지 털 이미지를 제시하였고, 동정의 오류를 다른 연구자들이 교정할 수 있는 시스템을 제공하는 데 큰 의미가 있다. 이 와 같은 우리의 시도가 향후 수행될 다른 식물상 연구뿐 만 아니라 식물을 주제로 하는 모든 연구 분야에 적용되 기를 기대하여 본다.

ORCID: Sungyu YANG https://orcid.org/0000-0001-50810296; Bo-Mi NAM https://orcid.org/0000-0002-6769-9317; JuEun JANG https://orcid.org/0000-0002-6229-8477; Mi-Jung CHOI https://orcid.org/0000-0002-4409-9765; Goya CHOI https://orcid.org/0000-0003-0530-0793; Kyong-Sook CHUNG https://orcid.org/0000-0002-4464-4698; Hyeok-Jae CHOI https://orcid.org/0000-0001-6315-0071

\section{Acknowledgments}

This research was funded by a grant from the Development of Sustainable Application for Standard Herbal Resources (KSN2012320) from the Korea Institute of Oriental Medicine (KIOM), South Korea.

\section{Conflict of Interest}

The authors declare that there are no conflicts of interest.

\section{Literature Cited}

Chilgok-gun. 2019. Natural environment of Chilgok-gun. Retrieved Sep. 2, 2019, available from http://www.chilgok.go.kr/07about/01_03.jsp.

Chung, G. Y., K. S. Chang, J.-M. Chung, H. J. Choi, W.-K. Paik and J.-O. Hyun. 2017. A checklist of endemic plants on the Korean Peninsula. Korean Journal of Plant Taxonomy 47: 264-288. (in Korean)

Compton, J. A., A. Culham and S. L. Jury. 1998. Reclassification of Actaea to include Cimicifuga and Souliea (Ranunculaceae): phylogeny inferred from morphology, nrDNA ITS, and cpDNA trnL-F sequence variation. Taxon 47: 593-634.

Funk, V. 2003. The importance of herbaria. Plant Science Bulletin 49: 94-95.

Han, K., S. So, C.-H. Lee and M. Kim. 2009. Taxonomy of the genus Scrophularia (Scrophulariaceae) in Korea. Korean Journal of Plant Taxonomy 39: 237-246. (in Korean)

Hwang, S. H., J. W. Lee, E. H. La and J. K. Ahn. 2020. Flora of the vascular plants of the Baekdudaegan conservation area: Deok-chi to Yuk-sim-nyeong. Korean Journal of Plant Taxonomy 50: 56-79. (in Korean)

Im, H.-T. 1999. Flora of Korea and the role of floristic study. Korean Journal of Plant Taxonomy 29: 275-284. (in Korean)

Jang, H. D., T. H. Kim and B. U. Oh. 2011. A taxonomic review of Scrophularia kakudensis Franch. and its relatives. Korean Journal of Plant Taxonomy 41: 345-352. (in Korean)

Jang, S. Y. 2010. A phylogenetic study of the genus Scrophularia in Korea. MS thesis, Yeongnam University, Gyeongsan, Korea. (in Korean)

Kang, E. S., S.-R. Lee, S. H. Oh, D.-K. Kim, S.-Y. Jung and D. C. Son. 2020. Comprehensive review about alien plants in Korea. Korean Journal of Plant Taxonomy 50: 89-119. (in Korean)

Kim, H.-W., E.-M. Sun, K.-H. Lee, J. S. Lee, B. K. Park, T.-Y. Choi and S.-R. Lee. 2020. Floristic study of Hwangmaesan Mt. Korean Journal of Plant Taxonomy 50: 199-226. (in Korean)

Kim, J.-S., B.-C. Lee, J.-M. Chung and J.-H. Pak. 2005. Flora and phytogeography on Mt. Deokhang (Gangwon-do), limestone area in Korea. Korean Journal of Plant Taxonomy 35: $337-$ 364. (in Korean)

Kim, K. J. and J. Y. Eo. 1998. Flora of Palgong Mountain (Daegu, Gunwi) and nearby mountains. 2nd National Natural Environment Survey: Daegu, Gunwi (10-17) natural environment. 
Ministry of Environment, Gwacheon. Pp. 127-189.

Kim, W. H., K. H. Bae, H. J. Cho and S. C. Hong. 1990. Forest vegetation and flora of Mt. Kasan: phytosociological analysis. Journal of Korean Forest Society 79: 42-55. (in Korean)

Korea National Arboretum. 2008. Rare Plants Data Book in Korea. Geobook Publishing Co., Pocheon, 332 pp. (in Korean)

Korea National Arboretum. 2016. Distribution Maps of Vascular Plants in Korea. Korea National Arboretum, Pocheon, 809 pp. (in Korean)

Korea National Arboretum. 2017. Checklist of Vascular Plants in Korea. Korea National Arboretum, Pocheon, 1,000 pp. (in Korean)

Korean Journal of Plant Taxonomy. 2020. Retrieved Aug. 11, 2020, available from https://www.e-kjpt.org/.

Lee, H.-W., M. G. Chung, Y. Suh and C.-W. Park. 2000. Allozyme variation and genetic relationships among species of Cimicifuga (Ranunculaceae) from Korea. International Journal of Plant Sciences 16: 413-423.

Lee, T. B. 1980. Illustrations of Flora of Korea. Hyangmunsa, Seoul, 990 pp. (in Korean)

Lee, W. T. 1996. Coloured Standard Illustrations of Korean Plants. Academy Publishing Co., Seoul, 624 pp. (in Korean)

Lee, Y. N. 1969. Swamp plants on Mt. Dae-Am in the central part of Korea. Korean Journal of Plant Taxonomy 1: 7-14. (in Korean)

Lee, Y. N. 1974. New taxa on Korean flora (1). Korean Journal of Plant Taxonomy 17: 33-35.

Nakai, T. 1909. Flora Koreana. Pars prima. Journnal College of Science, Imperial University of Tokyo 26: 1-304.

Nakai, T. 1938. Notulae ad Plantas Asiae Orientalis (V). Journal of Japanese Botany 14: 631-637.

National Institute of Biological Resources. 2012. Red Data Book of Endangered Vascular Plants in Korea. National Institute of Biological Resources, Incheon, 392 pp. (in Korean)

National Institute of Ecology. 2018. Floristic Target Species (FT species) in Korea. National Institute of Ecology, Seocheon, 728 pp. (in Korean)
Park, B. K., D. C. Son and S. C. Ko. 2020. The flora of vascular plants in Gibaeksan Mt. County Park and mountains neighboring the park. Korean Journal of Plant Taxonomy 50: 166198. (in Korean)

Park, S. H. 2009. New Illustrations and Photographs of Naturalized Plants of Korea. Ilchokak Publishing, Seoul, 575 pp. (in Korean)

Park, S. J., O. Son and E. M. Lee. 2015. The conservation and flora of Mt. Palgong (wetland, naturalized species). Nature Conservation 171: 21-28. (in Korean)

Son, O., S.-W. Son, G.-U. Suh and S. Park. 2015. Natural hybridization of Iris species in Mt. Palgong-san, Korea. Korean Journal of Plant Taxonomy 45: 243-253. (in Korean)

Suarez, A. V. and N. D. Tsutsui. 2004. The value of museum collections for research and society. BioScience 54: 66-74.

Sun, E.-M., H.-D. Son, S.-B. Park, U-N. Kim and H.-T. Im. 2020. Floristic study of Ibamsan Mt. and Baekamsan Mt. Korean Journal of Plant Taxonomy 50: 27-55. (in Korean)

The Angiosperm Phylogeny Group, M. W. Chase, M. J. M. Christenhusz, M. F. Fay, J. W. Byng, W. S. Judd, D. E. Soltis, D. J. Mabberley, A. N. Sennikov, P. S. Soltis and P. F. Stevens. 2016. An update of the Angiosperm Phylogeny Group classification for the orders and families of flowering plants: APG IV. Botanical Journal of the Linnean Society 181: 1-20.

The Plant List. 2013. Version 1.1. Retrieved Jan 1, 2020, available from http://www.theplantlist.org/.

The Pteridophyte Phylogeny Group. 2016. A community-derived classification for extant lycophytes and ferns. Journal of Systematics and Evolution 54: 563-603.

Yang, S., H.-D. Jang, B. M. Nam, G. Y. Chung, R.-Y. Lee, J.-H. Lee and B.-U. Oh. 2015. A florisitic study of Ulleungdo Island in Korea. Korean Journal of Plant Taxonomy 45: 192-212. (in Korean)

Zhao, Y., H. J. Noltie and B. Mathew. 2000. Iridaceae. In Flora of China, Vol. 24. Wu, Z. Y., P. H. Raven and D. Y. Hong (eds.), Science Press, Beijing and Missouri Botanical Garden Press, St. Louis, MO. Pp 297-313. 


\title{
온라인 생물정보 플랫폼에 기반한 가산의 관속식물목록
}

\author{
양선규 $^{\dagger} \cdot$ 남보미 $^{1 \dagger} \cdot$ 장주은 ${ }^{2} \cdot$ 최미정 ${ }^{2} \cdot$ 최고야 · 정경숙 ${ }^{3} \cdot$ 최혁재 ${ }^{2 *}$
}

한국한의학연구원 한약자원연구센터, ${ }^{1}$ 한국생명공학연구원 해외생물소재센터

2창원대학교 생물학화학융합학부, ${ }^{3}$ 중원대학교 제약공학과

적 요: 경상북도 칠곡군에 위치한 가산 $(902 \mathrm{~m})$ 의 관속식물상을 밝히고 주요 식물들의 분포를 파악하기 위 하여 2014년부터 2015년까지 10회의 현지조사를 수행하였다. 현지조사 결과 가산의 관속식물 81과 212속 289 종 8 아종 20 변종 5 품종으로 총 322 분류군의 분포를 확인하였다. 조사된 분류군 중 한반도 특산식물은 14 분류 군, 희귀식물은 6 분류군이 확인되었고 식물구계학적 특정식물종은 48 분류군이었으며 귀화식물은 14 분류군으로 나타났다. 조사지역은 세잎승마와 노랑무늬붓꽃의 분포한계 지역이었으며 가산산성 일대에서 금붓꽃과 노랑무늬 붓꽃의 잡종개체군을 확인하였다. 조사지역에서 수집된 관속식물 증거표본 325 점은 한국한의학연구원 한약표준 표본관에 보관하였고 디지털 이미지로 만들어 https:/oasis.kiom.re.kr/herblib/에 공유하였다. 본 연구는 국내 식물 상 연구분야에서 증거표본의 디지털 이미지를 공유한 최초의 연구로서 큰 의미를 갖는다.

주요어: 식물상, 가산, 관속식물, 증거표본, 온라인플랫폼 


\section{Appendix 1. List of vascular plants in Gasan Mt. including voucher specimens.}

[Abbreviation] E, endemic plants; CR, critical endangered plants; VU, vulnerable plants; LC, least concerned plants; I-V, degree of floristic target species; N, naturalized alien plants, Cult.: cultivated plants.

\section{Selaginellaceae 부처손과}

1. Selaginella rossii (Baker) Warb. 구실사리; KIOM202001023139

2. Selaginella tamariscina (P. Beauv.) Spring 바위손(III); KIOM202001023140

\section{Dennstaedtiaceae 잔고사리과}

3. Dennstaedtia hirsuta (Sw.) Mett. ex Miq. 잔고사리; KIOM202001023204

4. Dennstaedtia wilfordii (T.Moore) Christ 황고사리; KIOM202001023205

\section{Aspleniaceae 꼬리고사리과}

5. Asplenium incisum Thunb. 꼬리고사리; KIOM202001023024

\section{Woodsiaceae 우드풀과}

6. Woodsia manchuriensis Hook. 만주우드풀; KIOM202001023192

\section{Athyriaceae 개고사리과}

7. Anisocampium niponicum (Mett.) Y. C. Liu, W. L. Chiou \& M. Kato 개고사리; KIOM202001023189

8. Athyrium yokoscense (Franch. \& Sav.) Christ 뱀고사리; KIOM202001023190

9. Deparia coreana (Christ) M. Kato 곱새고사리; KIOM202001023191

\section{Dryopteridaceae 관중과}

10. Dryopteris chinensis (Baker) Koidz. 가는잎족제비고사리; KIOM202001023072

11. Dryopteris crassirhizoma Nakai 관중; KIOM202001023073

12. Dryopteris lacera (Thunb.) Kuntze 비늘고사리; KIOM202001023074

13. Dryopteris uniformis (Makino) Makino 곰비늘고사리; KIOM202001023075

\section{Davalliaceae 넉줄고사리과}

14. Davallia mariesii T. Moore ex Baker 넉줄고사리; KIOM202001023043

Pinaceae 소나무과

\author{
15. Larix kaempferi (Lamb.) Carrière 일본잎갈나무; \\ KIOM202001023170
}

\section{Schisandraceae 오미자과}

16. Schisandra chinensis (Turcz.) Baill. 오미자(II); KIOM202001023184

\section{Aristolochiaceae 쥐방울덩굴과}

17. Aristolochia contorta Bunge 쥐방울덩굴

18. Asarum patens (Yamaki) Yamaki ex Y. N. Lee 금오족도 리풀(E); KIOM202001023224

19. Asarum sieboldii Miq. 족도리풀; KIOM202001023225

\section{Magnoliaceae 목련과}

20. Magnolia sieboldii K. Koch 함박꽃나무(II); KIOM202001023076

\section{Lauraceae 녹나무과}

21. Lindera erythrocarpa Makino 비목나무(I); KIOM202001023049

\section{Araceae 천남성과}

22. Arisaema amurense Maxim. 둥근잎천남성; KIOM202001023238

23. Arisaema serratum (Thunb.) Schott 점박이천남성; KIOM202001023239

\section{Dioscoreaceae 마과}

24. Dioscorea japonica Thunb. 참마

25. Dioscorea nipponica Makino 부채마

\section{Melanthiaceae 멜란티움과}

26. Paris verticillata M. Bieb. 삿갓나물; KIOM202001023103

27. Veratrum maackii Regel 긴잎여로(III); KIOM202001023111

28. Veratrum versicolor f. viride Nakai 푸른여로; KIOM202001023288

29. Veratrum versicolor Nakai 흰여로; KIOM202001023112

\section{Colchicaceae 콜키쿰과}

30. Disporum smilacinum A. Gray 애기나리; KIOM202001023097

31. Disporum viridescens (Maxim.) Nakai 큰애기나리; KIOM202001023098 


\section{Smilacaceae 청미래덩굴과}

32. Smilax nipponica Miq. 선밀나물; KIOM202001023109

33. Smilax sieboldii Miq. 청가시덩굴; KIOM202001023110

\section{Liliaceae 백합과}

34. Amana edulis (Miq.) Honda 산자고; KIOM202001023287

35. Lilium tsingtauense Gilg 하늘말나리; KIOM202001023100

36. Lloydia triflora (Ledeb.) Baker 나도개감채(II, LC);

KIOM202001023102, KIOM202001023317

\section{Orchidaceae 난초과}

37. Cephalanthera longibracteata Blume 은대난초; KIOM202001023042

38. Cypripedium macranthos Sw. 복주머니란

39. Platanthera hologlottis Maxim. 흰제비란

\section{Iridaceae 붓꽃과}

40. Iris domestica (L.) Goldblatt \& Mabb. 범부채

41. Iris hybrid plant; KIOM202001023292

42. Iris minutoaurea Makino 금붓꽃(I, VU); KIOM202001023293

43. Iris odaesanensis Y. N. Lee 노랑무늬붓곷(E, IV, 2, VU); KIOM202001023294

44. Iris rossii Baker 각시붓꽃; KIOM202001023141

45. Iris ruthenica Ker Gawl. 솔붓꽃

46. Iris sanguinea Donn ex Hornem. 붓꽃

\section{Asphodelaceae 아스포델과}

47. Hemerocallis hakuunensis Nakai 백운산원추리(E); KIOM202001023099, KIOM202001023286

\section{Amaryllidaceae 수선화과}

48. Allium monanthum Maxim. 달래; KIOM202001023285

Asparagaceae 아스파라거스과

49. Asparagus schoberioides Kunth 비짜루; KIOM202001023096

50. Barnardia japonica (Thunb.) Schult. \& Schult. f. 무릇; KIOM202001023107

51. Liriope spicata Lour. 개맥문동; KIOM202001023101

52. Maianthemum japonicum (A.Gray) LaFrankie 풀솜대; KIOM202001023108

53. Polygonatum involucratum (Franch. \& Sav.) Maxim. 용둥 굴레; KIOM202001023104

54. Polygonatum lasianthum Maxim. 죽대; KIOM202001023105

55. Polygonatum odoratum var. pluriflorum (Miq.) Ohwi 둥굴 레; KIOM202001023106

\section{Commelinaceae 닭의장풀과}

56. Commelina communis L. 닭의장풀; KIOM202001023052

Juncaceae 골풀과

57. Juncus decipiens (Buchenau) Nakai 골풀; KIOM202001022998

58. Luzula capitata (Miq. ex Franch. \& Sav.) Kom. 꿩의밥; KIOM202001022999

\section{Cyperaceae 사초과}

59. Carex capillacea Boott 잔솔잎사초; KIOM202001023145

60. Carex hakonensis Franch. \& Sav. 애기바늘사초; KIOM202001023146

61. Carex japonica Thunb. 개찌버리사초; KIOM202001023147

62. Carex lanceolata Boott 그늘사초; KIOM202001023148

63. Carex leiorhyncha C. A. Mey. 산괭이사초; KIOM202001023149

64. Carex mitrata Franch. 청사초(I); KIOM202001023144

65. Carex miyabei Franch. 융단사초; KIOM202001023150

66. Carex neurocarpa Maxim. 괭이사초; KIOM202001023296

67. Carex onoei Franch. \& Sav. 바늘사초(II); KIOM202001023297

68. Carex siderosticta Hance 대사초; KIOM202001023298

69. Carex ciliatomarginata Nakai 털대사초; KIOM202001023295

70. Scirpus wichurae Boeckeler 방울고랭이; KIOM202001023299

\section{Poaceae 벼과}

71. Arundinella hirta var. ciliata (Thunb.) Koidz. 털새; KIOM202001023120

72. Bromus remotiflorus (Steud.) Ohwi 꼬리새; KIOM202001023121

73. Calamagrostis arundinacea (L.) Roth 실새풀; KIOM202001023122

74. Cymbopogon goeringii (Steud.) A. Camus 개솔새

75. Dactylis glomerata L. 오리새(N); KIOM202001023123

76. Diarrhena mandshurica Maxim. 껍질용수염; KIOM202001023124

77. Digitaria ciliaris (Retz.) Koeler 바랭이

78. Digitaria radicosa (J. Presl) Miq. 좀바랭이

79. Dimeria ornithopoda Trin. 잔디바랭이

80. Echinochloa crus-galli (L.) P. Beauv. 돌피; KIOM202001023125

81. Eriochloa villosa (Thunb.) Kunth 나도개피; KIOM202001023126

82. Festuca arundinacea Schreb. 큰김의털(N); KIOM202001023127 
83. Glyceria leptolepis Ohwi 왕미꾸리광이(II); KIOM202001023291

84. Miscanthus sacchariflorus (Maxim.) Hack. 물억새; KIOM202001023128

85. Miscanthus sinensis f. gracillimus (Hitchc.) Ohwi 가는잎 억새; KIOM202001023129

86. Oplismenus burmannii (Retz.) P. Beauv. 민주름조개풀; KIOM202001023130

87. Panicum dichotomiflorum Michx. 미국개기장 $(\mathrm{N})$; KIOM202001023131

88. Pennisetum alopecuroides (L.) Spreng. 수크령; KIOM202001023132

89. Poa anпиa L. 새포아풀; KIOM202001023133

90. Setaria faberi R. A. W. Herrm. 가을강아지풀; KIOM202001023134

91. Spodiopogon sibiricus Trin. 큰기름새; KIOM202001023135

92. Stipa pekinensis Hance 나래새; KIOM202001023136

\section{Papaveraceae 양귀비과}

93. Chelidonium asiaticum (H. Hara) Krahulc. 애기똥풀; KIOM202001023183

94. Corydalis heterocarpa Siebold \& Zucc. 염주괴불주머니

95. Corydalis heterocarpa var. japonica (Franch. \& Sav.) Ohwi 갯괴불주머니

96. Corydalis namdoensis B. U. Oh \& J. G. Kim 남도현호색 (E, III); KIOM202001023315

97. Corydalis remota Fisch. ex Maxim. 현호색; KIOM202001023271

98. Corydalis speciosa Maxim. 산괴불주머니; KIOM202001023272

99. Corydalis turtschaninovii Besser 조선현호색; KIOM202001023273

\section{Lardizabalaceae 으름덩굴과}

100. Akebia quinata (Houtt.) Decne. 으름덩굴; KIOM202001023196

\section{Ranunculaceae 미나리아재비과}

101. Aconitum jaluense Kom. 투구꽃(I); KIOM202001023080 102. Actaea asiatica H. Hara 노루삼(III); KIOM202001023081 103. Adonis amurensis Regel \& Radde 복수초; KIOM202001023082

104. Anemone raddeana Regel 꿩의바람꽃; KIOM202001023283

105. Cimicifuga heracleifolia var. bifida Nakai 세잎승마(E, IV, VU); KIOM202001023083

106. Clematis apiifolia DC. 사위질빵; KIOM202001023084
107. Clematis patens C. Morren \& Decne. 큰곷으아리(I); KIOM202001023085

108. Clematis terniflora var. mandshurica (Rupr.) Ohwi 으아 리; KIOM202001023086

109. Clematis trichotoma Nakai 할미밀망(E); KIOM202001023087

110. Eranthis stellata Maxim. 너도바람꽃(III, LC); KIOM202001023088

111. Pulsatilla cernua var. koreana (Yabe ex Nakai) Y. N. Lee 할미꽃

112. Ranunculus japonicus Thunb. 미나리아재비; KIOM202001023089

113. Ranunculus tachiroei Franch. \& Sav. 개구리미나리; KIOM202001023284

114. Thalictrum aquilegiifolium var. sibiricum Regel \& Tiling 꿩의다리; KIOM202001023090

115. Thalictrum minus subsp. thunbergii (DC.) Vorosch. 좀뀡 의다리; KIOM202001023091

\section{Haloragaceae 개미탑과}

116. Gonocarpus micranthus Thunb. 개미탑

\section{Saxifragaceae 범의귀과}

117. Astilbe koreana (Kom.) Nakai 숙은노루오줌; KIOM202001023289

118. Astilbe rubra Hook. f. \& Thomson 노루오줌; KIOM202001023114

119. Chrysosplenium flagelliferum F. Schmidt 애기괭이눈; KIOM202001023115

120. Chrysosplenium pilosum var. sphaerospermum (A.

Terracc.) H. Hara 흰갱이눈(E, II); KIOM202001023290

\section{Crassulaceae 돌나물과}

121. Sedum kamtschaticum Fisch. 기린초; KIOM202001023056

122. Sedum polytrichoides Hemsl. 바위채송화; KIOM202001023057

\section{Vitaceae 포도과}

123. Ampelopsis brevipedunculata f. ciliata (Nakai) T. B. Lee 털개머루; KIOM202001023262

124. Ampelopsis glandulosa var. brevipedunculata (Maxim.) Momiy. 개머루; KIOM202001023261

125. Parthenocissus tricuspidata (Siebold \& Zucc.) Planch. 담 쟁이덩굴; KIOM202001023263

126. Vitis flexuosa Thunb. 새머루

\section{Fabaceae 콩과}

127. Albizia julibrissin Durazz. 자귀나무 
128. Amphicarpaea bracteata subsp. edgeworthii (Benth.) H. Ohashi 새콩; KIOM202001023244

129. Campylotropis macrocarpa (Bunge) Rehder 꽃싸리(IV); KIOM202001023245

130. Crotalaria sessiliflora L. 활나물

131. Gleditsia japonica Miq. 주엽나무

132. Gleditsia sinensis Lam. 조각자나무

133. Glycine max subsp. soja (Siebold \& Zucc.) H. Ohashi 돌콩

134. Hylodesmum podocarpum (DC.) H. Ohashi \& R. R. Mill 개도둑놈의갈고리

135. Hylodesmum podocarpum subsp. oxyphyllum (DC.) H.

Ohashi \& R. R. Mill 도둑놈의갈고리; KIOM202001023246

136. Indigofera kirilowii Maxim. ex Palib. 땅비싸리; KIOM202001023247

137. Kummerowia striata (Thunb.) Schindl. 매듭풀

138. Lathyrus davidii Hance 활량나물; KIOM202001023248

139. Lespedeza $\times$ nakaii T. B. Lee 꽃참싸리; KIOM202001023249

140. Lespedeza $\times$ schindleri T. B. Lee 잡싸리; KIOM202001023250

141. Lespedeza bicolor Turcz. 싸리

142. Lespedeza cuneata (Dum. Cours.) G. Don 비수리

143. Lespedeza cyrtobotrya Miq. 참싸리; KIOM202001023251

144. Lespedeza maximowiczii C. K. Schneid. 조록싸리; KIOM202001023252

145. Lespedeza maximowiczii var. tomentella Nakai 털조록싸 리; KIOM202001023253

146. Lespedeza thunbergii subsp. formosa (Vogel) H. Ohashi 풀싸리; KIOM202001023314

147. Lespedeza tomentosa (Thunb.) Siebold ex Maxim. 개싸리

148. Lespedeza virgata (Thunb.) DC. 좀싸리

149. Pueraria montana var. lobata (Willd.) Sanjappa \&

Pradeep 칡

150. Robinia pseudoacacia L. 아까시나무(N); KIOM202001023254

151. Sophora flavescens Aiton 고삼

152. Vicia amurensis Oett. 벌완두

153. Vicia chosenensis Ohwi 노랑갈퀴(E, III); KIOM202001023255

154. Vicia cracca L. 등갈퀴나물

155. Vicia japonica A. Gray 넓은잎갈퀴

156. Vicia nipponica Matsum. 네잎갈퀴나물; KIOM202001023256

157. Vicia pseudo-orobus Fisch. \& C. A. Mey. 큰등갈퀴

158. Vicia ramuliflora (Maxim.) Ohwi 큰네잎갈퀴

159. Vicia unijuga A. Braun 나비나물; KIOM202001023257

160. Vicia venosa var. cuspidata Maxim. 광릉갈퀴;

KIOM202001023258
161. Wisteria floribunda (Willd.) DC. 등(IV, Cult.); KIOM202001023259

Polygalaceae 원지과

162. Polygala japonica Houtt. 애기풀

\section{Rosaceae 장미과}

163. Agrimonia coreana Nakai 산짚신나물;

$$
\text { KIOM202001023206 }
$$

164. Agrimonia pilosa Ledeb. 짚신나물; KIOM202001023207

165. Aruncus dioicus (Walter) Fernald 눈개승마(III); KIOM202001023208

166. Duchesnea indica (Jacks.) Focke 뱀딸기; KIOM202001023209

167. Geum aleppicum Jacq. 큰뱀무; KIOM202001023210 168. Potentilla ancistrifolia var. dickinsii (Franch. \& Sav.)

Koidz. 돌양지꽃(II); KIOM202001023211

169. Potentilla chinensis Ser. 딱지꽃

170. Potentilla fragarioides L. 양지꽃; KIOM202001023302

171. Potentilla rosulifera H. Lév. 민눈양지꽃(II);

KIOM202001023212

172. Prunus persica (L.) Batsch 복사나무; KIOM202001023303

173. Prunus serrulata f. spontanea (E.H.Wilson) Chin S.

Chang 벚나무

174. Prunus serrulata var. pubescens (Makino) Nakai 잔털벚 나무; KIOM202001023304

175. Prunus verecunda (Koidz.) Koehne 개벚나무

176. Rosa multiflora Thunb. 찔레꽃; KIOM202001023305

177. Rubus crataegifolius Bunge 산딸기; KIOM202001023213

178. Rubus parvifolius L. 멍석딸기

179. Rubus phoenicolasius Maxim. 곰딸기; KIOM202001023214

180. Rubus pungens Cambess. 줄딸기; KIOM202001023306

181. Sanguisorba officinalis L. 오이풀; KIOM202001023215

182. Sanguisorba officinalis var. longifolia (Bertol.) T. T. Yu \& C. L. Li 긴오이풀

183. Sorbus alnifolia (Siebold \& Zucc.) K. Koch 팥배나무; KIOM202001023216

184. Spiraea prunifolia var. simpliciflora (Nakai) Nakai 조팝 나무; KIOM202001023307

185. Stephanandra incisa (Thunb.) Zabel 국수나무; KIOM202001023217

\section{Ulmaceae 느릅나무과}

186. Ulmus davidiana var. japonica (Rehder) Nakai 느릅나무 187. Ulmus pumila L. 비술나무

188. Zelkova serrata (Thunb.) Makino 느티나무 


\section{Elaeagnaceae 보리수나무과}

189. Elaeagnus umbellata Thunb. 보리수나무; KIOM202001023137

\section{Cannabaceae 삼과}

190. Celtis aurantiaca Nakai 산팽나무

191. Celtis biondii Pamp. 폭나무

192. Celtis sinensis Pers. 팽나무

193. Humulus scandens (Lour.) Merr. 환삼덩굴

\section{Moraceae 뽛나무과}

194. Broussonetia hanjiana M. Kim 닥나무

195. Broussonetia papyrifera (L.) L'Hér. ex Vent. 꾸지나무; KIOM202001023142

196. Morus indica L. 산뽕나무; KIOM202001023143

\section{Urticaceae 쐐기풀과}

197. Boehmeria japonica (L. f.) Miq. 왜모시풀; KIOM202001023176

198. Boehmeria platanifolia (Franch. \& Sav.) C. H. Wright 개 모시풀; KIOM202001023316, KIOM202001023177

199. Boehmeria spicata (Thunb.) Thunb. 좀깨잎나무; KIOM202001023178

200. Pilea mongolica Wedd. 모시물통이; KIOM202001023179

\section{Fagaceae 참나무과}

201. Quercus acutissima Carruth. 상수리나무; KIOM202001023233

202. Quercus aliena Blume 갈참나무; KIOM202001023234

203. Quercus aliena var. acutiserrata Maxim. 졸갈참나무

204. Quercus dentata Thunb. 떡갈나무; KIOM202001023235

205. Quercus mongolica var. crispula (Blume) H. Ohashi 물 참나무

206. Quercus serrata Murray 졸참나무; KIOM202001023236

207. Quercus variabilis Blume 굴참나무; KIOM202001023237

\section{Juglandaceae 가래나무과}

208. Platycarya strobilacea Siebold \& Zucc. 굴피나무

\section{Betulaceae 자작나무과}

209. Alnus firma Siebold \& Zucc. 사방오리 210. Alnus hirsuta (Spach) Rupr. 물오리나무; KIOM202001023201

211. Alnus japonica var. rufa Nakai 털오리나무

212. Betula davurica Pall. 물박달나무

213. Betula platyphylla Sukaczev 자작나무

214. Carpinus cordata Blume 까치박달

215. Carpinus laxiflora (Siebold \& Zucc.) Blume 서어나무; KIOM202001023202
216. Carpinus tschonoskii Maxim. 개서어나무

217. Carpinus turczaninowii Hance 소사나무

218. Corylus heterophylla Fisch. ex Trautv. 개암나무; KIOM202001023203

\section{Celastraceae 노박덩굴과}

219. Celastrus orbiculatus Thunb. 노박덩굴; KIOM202001023046

220. Euonymus alatus f. ciliatodentatus (Franch. \& Sav.)

Hiyama 회잎나무; KIOM202001023047

221. Euonymus hamiltonianus Wall. 참빗살나무;

KIOM202001023276

222. Euonymus sachalinensis (F. Schmidt) Maxim. 회나무(I); KIOM202001023048

\section{Hypericaceae 물레나물과}

223. Hypericum ascyron L. 물레나물; KIOM202001023077 224. Hypericum erectum Thunb. 고추나물; KIOM202001023078

225. Hypericum laxum (Blume) Koidz. 좀고추나물; KIOM202001023281

\section{Linaceae 아마과}

226. Linum stelleroides Planch. 개아마

\section{Violaceae 제비꽃과}

227. Viola acuminata Ledeb. 졸방제비꽃; KIOM202001023218

228. Viola albida var. chaerophylloides (Regel) F. Maek. ex Hara 남산제비꽃; KIOM202001023220

229. Viola albida var. takahashii (Nakai) Nakai 단풍제비꽃; KIOM202001023219

230. Viola arcuata Blume 콩제비꽃; KIOM202001023312

231. Viola collina Besser 둥근털제비꽃; KIOM202001023221

232. Viola kamibayashii Nakai 광릉제비꽃

233. Viola keiskei Miq. 잔털제비꽃; KIOM202001023308

234. Viola lactiflora Nakai 흰젖제비꽃; KIOM202001023309 235. Viola mandshurica W. Becker 제비꽃; KIOM202001023310

236. Viola orientalis (Maxim.) W. Becker 노랑제비꽃(II); KIOM202001023222

237. Viola phalacrocarpa Maxim. 털제비꽃; KIOM202001023311

238. Viola rossii Hemsl. 고깔제비꽃

239. Viola seoulensis Nakai 서울제비꽃

240. Viola variegata Fisch. ex Link 알록제비꽃; KIOM202001023223 


\section{Salicaceae 버드나무과}

241. Salix babylonica L. 수양버들

242. Salix caprea L. 호랑버들; KIOM202001023113

243. Salix chaenomeloides Kimura 왕버들

244. Salix gilgiana Seem. 내버들

245. Salix pseudolasiogyne H. Lév. 능수버들

\section{Euphorbiaceae 대극과}

246. Acalypha australis L. 깨풀; KIOM202001023053

247. Euphorbia jolkinii Boiss. 암대극

248. Euphorbia pekinensis Rupr. 대극(I); KIOM202001023318

249. Euphorbia sieboldiana Morren \& Decne. 개감수;

KIOM202001023054

\section{Phyllanthaceae 여우주머니과}

250. Flueggea suffruticosa (Pall.) Baill. 광대싸리; KIOM202001023055

251. Phyllanthus ussuriensis Rupr. \& Maxim. 여우주머니

\section{Geraniaceae 쥐손이풀과}

252. Geranium koreanum Kom. 둥근이질풀(II); KIOM202001023226

253. Geranium koreanum var. hirsutum Nakai 털둥근이질풀

254. Geranium sibiricum L. 쥐손이풀

255. Geranium thunbergii Siebold ex Lindl. \& Paxton 이질풀

256. Geranium wilfordii Maxim. 세잎쥐손이; KIOM202001023227

\section{Onagraceae 바늘꽃과}

257. Circaea mollis Siebold \& Zucc. 털이슬; KIOM202001023092

258. Epilobium amurense subsp. cephalostigma (Hausskn.) C.

J. Chen \& Hoch \& P. H. Raven 돌바늘꽃

259. Ludwigia prostrata Roxb. 여뀌바늘

260. Oenothera biennis L. 달맞이꽃(N); KIOM202001023093

\section{Lythraceae 부처꽃과}

261. Lagerstroemia indica L. 배롱나무

\section{Staphyleaceae 고추나무과}

262. Staphylea bumalda DC. 고추나무; KIOM202001022997

\section{Anacardiaceae 옻나무과}

263. Acer palmatum Thunb. 단풍나무

264. Acer pictum subsp. mono (Maxim.) H. Ohashi 고로쇠나무 265. Acer pictum Thunb. 털고로쇠나무

266. Acer pseudosieboldianum var. ishidoyanum (Nakai) Uyeki 산단풍나무
267. Rhus chinensis Mill. 붉나무; KIOM202001023185

268. Toxicodendron trichocarpum (Miq.) Kuntze 개옻나무; KIOM202001023186

\section{Sapindaceae 무환자나무과 \\ Simaroubaceae 소태나무과}

269. Acer pseudosieboldianum (Pax) Kom. 당단풍나무; KIOM202001023051

270. Picrasma quassioides (D. Don) Benn. 소태나무

\section{Rutaceae 운향과}

271. Dictamnus dasycarpus Turcz. 백선(I);

$$
\text { KIOM202001023193 }
$$

272. Tetradium daniellii (Benn.) T.G.Hartley 쉬나무

273. Zanthoxylum piperitum (L.) DC. 초피나무; KIOM202001023194

274. Zanthoxylum schinifolium Siebold \& Zucc. 산초나무; KIOM202001023195

\section{Malvaceae 아욱과}

275. Corchoropsis tomentosa (Thunb.) Makino 수까치깨; KIOM202001023264

\section{Brassicaceae 십자화과}

276. Capsella bursa-pastoris (L.) Medik. 냉이; KIOM202001023171

277. Cardamine fallax (O. E. Schulz) Nakai 좁쌀냉이; KIOM202001023172

278. Cardamine leucantha (Tausch) O. E. Schulz 미나리냉이 ; KIOM202001023173

279. Rorippa indica (L.) Hiern 개갓냉이; KIOM202001023174

280. Thlaspi arvense L. 말냉이; KIOM202001023175

\section{Santalaceae 단향과}

281. Thesium chinense Turcz. 제비꿀

\section{Polygonaceae 마디풀과}

282. Aconogonon alpinum (All.) Schur 싱아; KIOM202001023059

283. Aconogonon divaricatum (L.) Nakai ex T.Mori 왜개싱아 284. Aconogonon microcarpum (Kitag.) H. Hara 참개싱아 285. Antenoron filiforme (Thunb.) Roberty \& Vautier 이삭여 뀌; KIOM202001023062

286. Bistorta manshuriensis Kom. 범꼬리(II); KIOM202001023060

287. Fallopia dentatoalata (F. Schmidt) Holub 큰닭의덩굴; KIOM202001023061

288. Persicaria debilis (Meisn.) H. Gross ex Mori 세뿔여뀌 
289. Persicaria lapathifolia (L.) Delarbre 흰여뀌

290. Persicaria nodosa (Pers.) Opiz 명아자여뀌

291. Persicaria odorata subsp. conspicua (Nakai) Yonek. 꽃 여뀌

292. Persicaria orientalis (L.) Spach 털여뀌; KIOM202001023063

293. Polygonum dissitiflorum Hemsl. 가시여뀌; KIOM202001023278

294. Polygonum posumbu Buch.-Ham. ex D. Don 장대여뀌; KIOM202001023064

295. Polygonum senticosum (Meisn.) Franch. \& Sav. 며느리 밑씻개; KIOM202001023279

296. Polygonum thunbergii Siebold \& Zucc. 고마리

297. Polygonum viscoferum var. robustum Makino 큰근근이 여뀌; KIOM202001023280

298. Reynoutria japonica Houtt. 호장근

299. Rumex acetosa L. 수영

300. Rumex acetosella L. 애기수영; KIOM202001023065

301. Rumex crispus L. 소리쟁이

302. Rumex japonicus Houtt. 참소리쟁이;

KIOM202001023066

303. Rumex obtusifolius L. 돌소리쟁이; KIOM202001023067

\section{Amaranthaceae 비름과}

304. Chenopodium giganteum D. Don 명아주

\section{Caryophyllaceae 석죽과}

305. Arenaria serpyllifolia L. 벼룩이자리; KIOM202001023163

306. Dianthus chinensis L. 패랭이꽃

307. Dianthus longicalyx Miq. 술패랭이꽃

308. Lychnis cognata Maxim. 동자꽃(II); KIOM202001023164

309. Pseudostellaria heterophylla (Miq.) Pax 개별꽃; KIOM202001023165

310. Pseudostellaria palibiniana (Takeda) Ohwi 큰개별꽃; KIOM202001023300

311. Silene baccifera (L.) Roth 덩굴별꽃

312. Silene firma f. pubescens (Makino) Ohwi \& Ohashi 털 장구채

313. Silene firma Siebold \& Zucc. 장구채; KIOM202001023166

314. Silene seoulensis Nakai 가는장구채; KIOM202001023167 315. Stellaria aquatica (L.) Scop. 쇠별꽃; KIOM202001023168 316. Stellaria media (L.) Vill. 별꽃; KIOM202001023169

\section{Hydrangeaceae 수국과}

317. Deutzia parviflora Bunge 말발도리(I); KIOM202001023116
318. Deutzia uniflora Shirai 매화말발도리(I); KIOM202001023117

319. Hydrangea serrata f. acuminata (Siebold \& Zucc.) E. H. Wilson 산수국; KIOM202001023118

320. Philadelphus tenuifolius Rupr. 얇은잎고광나무; KIOM202001023119

\section{Cornaceae 충충나무과}

321. Alangium platanifolium (Siebold \& Zucc.) Harms 박쥐나 무(DD); KIOM202001023095

322. Cornus controversa Hemsl. 층층나무; KIOM202001023242

323. Cornus macrophylla Wall. 곰의말채나무; KIOM202001023243

324. Cornus walteri Wangerin 말채나무

\section{Balsaminaceae 봉선화과}

325. Impatiens noli-tangere L. 노랑물봉선

326. Impatiens textorii Miq. 물봉선; KIOM202001023138

Primulaceae 앵초과

327. Androsace umbellata (Lour.) Merr. 봄맞이; KIOM202001023180

328. Lysimachia clethroides Duby 큰까치수염; KIOM202001023181

329. Lysimachia davurica Ledeb. 좁쌀풀(III); KIOM202001023182

\section{Symplocaceae 노린재나무과}

330. Symplocos sawafutagi Nagam. 노린재나무; KIOM202001023275

331. Symplocos tanakana Nakai 검노린재나무

\section{Styracaceae 때죽나무과}

332. Styrax japonicus Siebold \& Zucc. 때죽나무

333. Styrax obassis Siebold \& Zucc. 쪽동백나무; KIOM202001023058

\section{Actinidiaceae 다래나무과}

334. Actinidia arguta (Siebold \& Zucc.) Planch. ex Miq. 다래 ; KIOM202001023277

335. Actinidia polygama (Siebold \& Zucc.) Planch. ex Maxim. 개다래; KIOM202001023050

\section{Ericaceae 진달래과}

336. Chimaphila japonica Miq. 매화노루발; KIOM202001023044

337. Pyrola japonica Klenze ex Alef. 노루발; KIOM202001023045 
338. Rhododendron dauricum var. ciliatum (Nakai) E. H.

Wilson 털진달래

339. Rhododendron mucronulatum Turcz. 진달래; KIOM202001023313

340. Rhododendron schlippenbachii Maxim. 철쭉; KIOM202001023231

\section{Rubiaceae 꼭두서니과}

341. Galium kamtschaticum Steller ex Roem. \& Schult. 털둥 근갈퀴

342. Galium maximowiczii (Kom.) Pobed. 개갈퀴; KIOM202001023025

343. Galium pogonanthum Franch. \& Sav. 산갈퀴; KIOM202001023026

344. Galium verum subsp. asiaticum (Nakai) T. Yamaz. 솔나 물; KIOM202001023027

345. Paederia scandens var. velutina (Nakai) Nakai 털계요등 346. Rubia argyi (H. Lév. \& Vaniot) H. Hara ex Lauener 꼭 두서니; KIOM202001023028

347. Rubia chinensis Regel \& Maack 큰꼭두서니(II); KIOM202001023029

348. Rubia cordifolia L. 갈퀴꼭두서니

\section{Gentianaceae 용담과}

349. Gentiana scabra Bunge 용담

350. Gentiana squarrosa Ledeb. 구슬붕이

351. Gentiana triflora subsp. japonica (Kusn.) Vorosch. 과남풀

352. Gentiana zollingeri Fawc. 큰구슬붕이;

KIOM202001023187

353. Swertia pseudochinensis H. Hara 자주쓴풀; KIOM202001023188

\section{Apocynaceae 협죽도과}

354. Cynanchum ascyrifolium (Franch. \& Sav.) Matsum. 민백 미꽃(I); KIOM202001023094

355. Cynanchum atratum Bunge 백미꽃

356. Metaplexis japonica (Thunb.) Makino 박주가리

357. Vincetoxicum inamoenum Maxim. 선백미꽃

\section{Boraginaceae 지치과}

358. Brachybotrys paridiformis Maxim. ex Oliv. 당개지치(III); KIOM202001023228

359. Lithospermum erythrorhizon Siebold \& Zucc. 지치

360. Trigonotis icumae (Maxim.) Makino 덩굴꽃마리(I, LC); KIOM202001023229

361. Trigonotis peduncularis (Trevis.) Benth. ex Baker \& S.

Moore 꽃마리; KIOM202001023230

362. Trigonotis radicans (Turcz.) Steven 거센털꽃마리
363. Trigonotis radicans var. sericea (Maxim.) H. Hara 참꽃 마리

\section{Convolvulaceae 메꽃과}

364. Cuscuta japonica Choisy 새삼

365. Cuscuta pentagona Engelm. 미국실새삼(N); KIOM202001023071

\section{Solanaceae 가지과}

366. Lycium chinense Mill. 구기자나무

367. Physaliastrum japonicum (Franch. \& Sav.) Honda 가시 꽈리; KIOM202001022994

368. Physalis angulata L. 땅꽈리

369. Solanum japonense Nakai 좁은잎배풍등;

$$
\text { KIOM202001022995 }
$$

370. Solanum lyratum Thunb. 배풍등

371. Solanum nigrum L. 까마중; KIOM202001022996

\section{Oleaceae 물푸레나무과}

372. Fraxinus chinensis subsp. rhynchophylla (Hance) A. E. Murray 물푸레나무

373. Fraxinus sieboldiana Blume 쇠물푸레나무; KIOM202001023282

374. Ligustrum obtusifolium Siebold \& Zucc. 쥐똥나무; KIOM202001023079

375. Ligustrum obtusifolium subsp. microphyllum (Nakai) P.

S. Green 좀쥐똥나무

\section{Plantaginaceae 질경이과}

376. Plantago asiatica L. 질경이; KIOM202001023232

377. Plantago major var. japonica (Franch. \& Sav.) Miyabe 왕질경이

378. Veronica daurica Steven 구와꼬리풀(III); KIOM202001023268

379. Veronica persica Poir. 큰개불알풀 $(\mathrm{N})$; KIOM202001023270

380. Veronica pyrethrina Nakai 큰구와꼬리풀(E, III, DD); KIOM202001023269

\section{Scrophulariaceae 현삼과}

381. Scrophularia cephalantha Nakai 몽울토현삼(E); KIOM202001023267

\section{Lamiaceae 꿀풀과}

382. Agastache rugosa (Fisch. \& C. A. Mey.) Kuntze 배초향; KIOM202001023030

383. Ajuga spectabilis Nakai 자란초(E, II); KIOM202001023031 
384. Callicarpa dichotoma (Lour.) K. Koch 좀작살나무

385. Callicarpa japonica Thunb. 작살나무;

KIOM202001023069

386. Callicarpa japonica var. luxurians Rehder 왕작살나무

387. Clerodendrum trichotomum Thunb. 누리장나무; KIOM202001023070

388. Clinopodium chinense var. parviflorum (Kudô) H. Hara 층층이꽃

389. Clinopodium chinense var. shibetchense (H. Lév.) Koidz. 산층층이

390. Clinopodium micranthum (Regel) H. Hara 두메층층이; KIOM202001023032

391. Elsholtzia ciliata (Thunb.) Hyl. 향유; KIOM202001023033

392. Elsholtzia splendens Nakai ex F. Maek. 꽃향유; KIOM202001023034

393. Galeopsis bifida Boenn. 털향유

394. Isodon inflexus (Thunb.) Kudô 산박하; KIOM202001023035

395. Isodon japonicus (Burm.) H. Hara 방아풀

396. Lamium album subsp. barbatum (Siebold \& Zucc.)

Mennema 광대수염; KIOM202001023036

397. Leonurus japonicus Houtt. 익모초

398. Lycopus lucidus Turcz. ex Benth. 쉽싸리; KIOM202001023037

399. Meehania urticifolia (Miq.) Makino 벌깨덩굴; KIOM202001023038

400. Mentha canadensis L. 박하

401. Mosla dianthera (Buch.-Ham. ex Roxb.) Maxim. 쥐깨풀 402. Phlomoides umbrosa (Turcz.) Kamelin \& Makhm. 속단; KIOM202001023039

403. Prunella vulgaris subsp. asiatica (Nakai) H. Hara 꿀풀 404. Scutellaria pekinensis Maxim. 산골무꽃;

KIOM202001023040

405. Scutellaria pekinensis var. ussuriensis (Regel) Hand.-

Mazz. 호골무꽃; KIOM202001023041

\section{Phrymaceae 파리풀과}

406. Phryma leptostachya var. oblongifolia (Koidz.) Honda 파 리풀; KIOM202001023260

\section{Paulowniaceae 오동나무과}

407. Paulownia coreana Uyeki 오동나무(E); KIOM202001023266

\section{Orobanchaceae 열당과}

408. Euphrasia maximowiczii Wettst. ex Palib. 앉은좁쌀풀 409. Melampyrum roseum Maxim. 꽃며느리밥풀; KIOM202001023265
410. Phtheirospermum japonicum (Thunb.) Kanitz 나도송이풀

\section{Campanulaceae 초롱꽃과}

411. Adenophora divaricata Franch. \& Sav. 넓은잔대;

KIOM202001023240

412. Adenophora triphylla var. japonica (Regel) H. Hara 잔대 413. Campanula punctata Lam. 초롱꽃(I); KIOM202001023241

414. Lobelia sessilifolia Lamb. 숫잔대

415. Platycodon grandiflorus (Jacq.) A. DC. 도라지

\section{Asteraceae 국화과}

416. Achillea alpina L. 톱풀

417. Ainsliaea acerifolia Sch. Bip. 단풍취;

KIOM202001023000

418. Ambrosia artemisiifolia L. 돼지풀(N); KIOM202001023001

419. Artemisia aпnиa L. 개똥쑥

420. Artemisia indica Willd. 灸

421. Artemisia japonica Thunb. 제비쑥; KIOM202001023003

422. Artemisia keiskeana Miq. 맑은대쑥; KIOM202001023004

423. Artemisia montana (Nakai) Pamp. 산쑥

424. Artemisia rubripes Nakai 덤불쑥

425. Artemisia sacrorum var. iwayomogi (Kitam.) M. S. Park

\& G. Y. Chung 더위지기; KIOM202001023002

426. Artemisia stolonifera (Maxim.) Kom. 넓은잎외잎쑥;

KIOM202001023005

427. Aster ageratoides Turcz. 까실쑥부쟁이; KIOM202001023006

428. Aster incisus Fisch. 가새쑥부쟁이; KIOM202001023007 429. Aster meyendorffii (Regel \& Maack) Voss 개쑥부쟁이 430. Atractylodes ovata (Thunb.) DC. 삽주;

KIOM202001023009

431. Bidens bipinnata L. 도깨비바늘

432. Bidens parviflora Willd. 까치발

433. Carpesium abrotanoides L. 담배풀

434. Carpesium cernuum L. 좀담배풀; KIOM202001023010

435. Carpesium macrocephalum Franch. \& Sav. 여우오줌

436. Chrysanthemum indicum L. 감국

437. Cirsium japonicum var. maackii (Maxim.) Matsum. 엉겅 퀴; KIOM202001023011

438. Cirsium setidens (Dunn) Nakai 고려엉겅퀴(E, I); KIOM202001023012

439. Conyza canadensis (L.) Cronquist 망초(N); KIOM202001023013

440. Crepidiastrum chelidoniifolium (Makino) Pak \& Kawano 까치고들빼기; KIOM202001023014

441. Crepidiastrum denticulatum (Houtt.) Pak \& Kawano 이 고들빼기; KIOM202001023015 
442. Crepidiastrum sonchifolium (Bunge) Pak \& Kawano 고 들빼기

443. Dendranthema boreale (Makino) Ling ex Kitam. 산국; KIOM202001023016

444. Dendranthema zawadskii var. latilobum (Maxim.) Kitam. 구절초; KIOM202001023017

445. Eupatorium japonicum Thunb. 등골나물; KIOM202001023018

446. Eupatorium lindleyanum DC. 골등골나물

447. Eupatorium tripartitum (Makino) Murata \& H. Koyama 항등골나물

448. Farfugium japonicum (L.) Kitam. 털머위

449. Hemisteptia lyrata (Bunge) Fisch. \& C. A. Mey. 지칭개; KIOM202001023019

450. Hieracium umbellatum L. 조밥나물

451. Inula linariifolia Turcz. 가는금불초

452. Ixeridium dentatum (Thunb.) Tzvelev 씀바귀

453. Lactuca indica f. indivisa (Makino) Hara 가는잎왕고들 빼기

454. Lactuca indica L. 왕고들빼기

455. Lactuca raddeana Maxim. 산씀바귀;

KIOM202001023020

456. Leibnitzia anandria (L.) Turcz. 솜나물

457. Leontopodium leontopodioides (Willd.) Beauverd 들떡쑥 458. Picris hieracioides subsp. japonica (Thunb.) Hand.-Mazz.

쇠서나물

459. Pseudognaphalium affine (D. Don) Anderb. 떡쑥

460. Rhaponticum uniflorum (L.) DC. 뻐꾹채

461. Saussurea conandrifolia Nakai 담배취

462. Saussurea japonica (Thunb.) DC. 큰각시취

463. Saussurea seoulensis Nakai 분취

464. Scorzonera austriaca Willd. 멱쇠채

465. Senecio argunensis Turcz. 쑥방망이

466. Sigesbeckia glabrescens (Makino) Makino 진득찰

467. Sigesbeckia orientalis subsp. pubescens (Makino) Kitam.

털진득찰; KIOM202001023021

468. Solidago virgaurea subsp. asiatica Kitam. ex H. Hara 미 역취

469. Symphyotrichum pilosum (Willd.) G. L. Nesom 미국쑥부 쟁이 $(\mathrm{N}) ;$ KIOM202001023008

470. Syneilesis palmata (Thunb.) Maxim. 우산나물;

KIOM202001023022

471. Synurus excelsus (Makino) Kitam. 큰수리취; KIOM202001023023

472. Synurus palmatopinnatifidus (Makino) Kitam. 국화수리취 473. Taraxacum officinale aggr. F. H. Wigg. 서양민들레(N); KIOM202001023274

474. Xanthium strumarium L. 도고마리

\section{Adoxaceae 연복초과}

475. Sambucus williamsii Hance 딱총나무; KIOM202001023198

476. Viburnum opulus subsp. calvescens (Rehder) Sugim. 백 당나무(I); KIOM202001023301

\section{Caprifoliaceae 인동과}

477. Lonicera japonica Thunb. 인동덩굴

478. Lonicera praeflorens Batalin 올괴불나무; KIOM202001023197

479. Lonicera subsessilis Rehder 청괴불나무

480. Patrinia scabiosifolia Link 마타리

481. Patrinia villosa (Thunb.) Juss. 뚝갈; KIOM202001023068

482. Scabiosa comosa Fisch. ex Roem. \& Schult. 솔체꽃

483. Weigela florida (Bunge) A. DC. 붉은병꽃나무(II); KIOM202001023199

484. Weigela subsessilis (Nakai) L. H. Bailey 병꽃나무(E); KIOM202001023200

\section{Araliaceae 두릅나무과}

485. Aralia elata (Miq.) Seem. 두릅나무

486. Kalopanax septemlobus f. maximowiczii (Van Houtte) H. Ohashi 가는잎음나무

\section{Apiaceae 미나리과}

487. Angelica amurensis Schischk. 지리강활

488. Angelica anomala Avé-Lall. 개구릿대

489. Angelica decursiva (Miq.) Franch. \& Sav. 바디나물; KIOM202001023151

490. Angelica genuflexa Nutt. ex Torr. \& A. Gray 왜천궁(III); KIOM202001023152

491. Angelica gigas Nakai 참당귀

492. Bupleurum falcatum L. 시호

493. Bupleurum longiradiatum Turcz. 개시호(II); KIOM202001023153

494. Cryptotaenia japonica Hassk. 파드득나물

495. Cymopterus melanotilingia (H.Boissieu) C. Y. Yoon 큰참 나물(II); KIOM202001023154

496. Oenanthe javanica (Blume) DC. 미나리; KIOM202001023155

497. Osmorhiza aristata Makino \& Jabe 긴사상자; KIOM202001023156

498. Ostericum grosseserratum (Maxim.) Kitag. 신감채; KIOM202001023157

499. Ostericum sieboldii (Miq.) Nakai 묏미나리; KIOM202001023158

500. Peucedanum terebinthaceum (Fisch. ex Trevir.) Ledeb. 기름나물

501. Peucedanum terebinthaceum var. deltoideum (Makino ex

K. Yabe) Makino 산기름나물 
502. Pimpinella brachycarpa (Kom.) Nakai 참나물;

KIOM202001023159

503. Sanicula chinensis Bunge 참반디; KIOM202001023160

504. Sanicula tuberculata Maxim. 애기참반디(III);

KIOM202001023161

505. Torilis japonica (Houtt.) DC. 사상자;

KIOM202001023162 